\title{
Neutron-rich gamma-ray burst flows: dynamics and particle creation in neutron-proton collisions
}

\author{
H. B. J. Koers ${ }^{1,2}$ and D. Giannios ${ }^{3}$ \\ 1 Nikhef, PO Box 41882, 1009 DB Amsterdam, The Netherlands \\ e-mail: hkoers@nikhef.nl \\ 2 University of Amsterdam, Amsterdam, The Netherlands \\ 3 Max Planck Institute for Astrophysics, PO Box 1317, 85741 Garching, Germany
}

Received 28 March 2007 / Accepted 7 June 2007

\section{ABSTRACT}

\begin{abstract}
We consider gamma-ray burst outflows with a substantial neutron component that are either dominated by thermal energy (fireballs) or by magnetic energy. In the latter case, we focus on the recently introduced "AC" model which relies on magnetic reconnection to accelerate the flow and power the prompt emission. For both the fireball and the AC model, we investigate the dynamical importance of neutrons on the outflow. We study particle creation in inelastic neutron-proton collisions and find that in both models the resulting neutrino emission is too weak to be detectable. The inelastic collisions also produce $\gamma$-rays, which create pairs in interactions with soft photons carried with the flow. In magnetically driven outflows, the energy of these pairs is radiated away as synchrotron emission. The bulk of the emission takes place at a few hundred $\mathrm{keV}$, which makes it difficult to disentangle this signal from the prompt emission. In fireballs, however, pair cascading leads to the emission of $\gamma$-rays with observer energy in the range of 2-20 GeV and a fluence well above the GLAST threshold. Therefore this emission can be a useful diagnostic of the nature of the outflow.
\end{abstract}

Key words. gamma rays: bursts - magnetohydrodynamics (MHD) - neutrinos - radiation mechanisms: general

\section{Introduction}

In recent years there has been significant progress in our understanding of $\gamma$-ray bursts (GRBs). The observational connection between supernovae and GRBs and studies of GRB host galaxies provide compelling evidence for a connection between long GRBs and the death of massive stars (Van Paradijs et al. 2000; Woosley \& Bloom 2006). The general scenario for long GRBs (for recent reviews, see Piran 2004; Mészáros 2006) starts with core collapse of the massive star leading to the formation of a black hole surrounded by an accretion disk. The black hole accretion disk system powers a developing outflow along the rotational axis, which accelerates to a bulk Lorentz factor of a few hundred, transferring its energy to the baryons contained in the flow. Dissipation of energy in the outflow leads to the prompt $\gamma$-ray emission while the interaction of the outflow with the external medium results in the afterglow.

The nature of the relativistic outflow is currently one of the most important open questions regarding GRBs. The high Lorentz factor, required to match the inferred energy density of the source and the observed non-thermal character of the emission (the compactness problem; see e.g. Piran 2004), implies that the ratio of energy to rest mass of the flow must be very high. In the widely used fireball model (Cavallo \& Rees 1978; Goodman 1986; Paczyński 1986) the outflow is a photonelectron-positron plasma that is dominated by thermal energy and has a small baryonic load. Alternatively, the energy of the outflow may initially be dominated by Poynting flux (Usov 1992). Such outflows occur naturally when a magnetized accretion disk surrounds a black hole (Thompson 1994; Mészáros \& Rees 1997; Spruit et al. 2001; Van Putten \& Ostriker 2001;
Vlahakis \& Königl 2001; Drenkhahn \& Spruit 2002; Lyutikov \& Blandford 2003; Lyutikov 2006; Uzdensky \& MacFadyen 2006).

Neutrinos and $\gamma$-rays may be useful probes to differentiate between fireballs and Poynting-flux dominated (PFD) outflows. The internal shocks that are believed to accelerate electrons in the fireball model will also accelerate protons to very high energies, giving rise to neutrinos with energy $\gtrsim 100 \mathrm{TeV}$ through photopion production (Waxman \& Bahcall 1997). In the absence of a mechanism to accelerate protons to very high energies these neutrinos are not expected in PFD outflows. In this paper we consider neutron-proton $(n p)$ collisions in neutron-rich flows and address the question whether neutrinos and $\gamma$-rays created in these hadronic interactions can also be used to probe the nature of GRB outflows.

GRB outflows are expected to be neutron-rich. In GRB central engines, the competition of positron capture on neutrons and electron capture on protons favours a neutron-rich environment (Beloborodov 2003b; Pruet et al. 2003; Chen \& Beloborodov 2007). Nucleosynthesizing interactions reduce the number of free neutrons in the outflow, but a significant amount of neutrons remains in the flow until neutron decay becomes important (Beloborodov 2003b; Inoue et al. 2003). Deep in the outflow protons and neutrons are strongly coupled through nuclear scattering and behave as a single fluid that accelerates to high Lorentz factors. With increasing distance from the central engine the densities decrease until neutrons decouple and enter the coasting phase. Protons, being electromagnetically coupled to the flow, may be accelerated further. When the relative velocity between neutrons and protons is sufficiently high, inelastic $n p$ collisions are possible and lead to pion creation. The 
pions decay into $\gamma$-rays and neutrinos with observer energies in the $\sim 10-100 \mathrm{GeV}$ range. This mechanism has been investigated for fireballs (Derishev et al. 1999a; Bahcall \& Mészáros 2000; Mészáros \& Rees 2000; Belyanin et al. 2003; Razzaque \& Mészáros 2006) but, to the best of our knowledge, not for PFD flows.

The creation of secondary particles in inelastic $n p$ collisions can potentially be used to identify a substantial neutron component in GRB flows. Other ways to identify such a component that have been suggested in the literature are through signatures in the early afterglow of GRBs (Derishev et al. 1999b; Beloborodov 2003a; Fan et al. 2005), ultraviolet flashes generated in internal shocks in neutron-rich flows (Fan \& Wei 2004), and observational signatures of a two-component jet that may be associated with neutron-rich MHD flows (Vlahakis et al. 2003; Peng et al. 2005).

In this work we consider the "AC" model as a specific model for PFD outflows. In this model the magnetic field configuration is similar to that produced by an inclined rotator (Coroniti 1990; Lyubarsky \& Kirk 2001) with field lines changing polarity on a scale $\lambda \simeq 2 \pi c / \Omega$, where $\Omega$ denotes the angular frequency of the rotator. This model was recently discussed in connection to GRBs in a series of papers (Spruit et al. 2001; Drenkhahn 2002; Drenkhahn \& Spruit 2002; Giannios \& Spruit 2005; Giannios 2006), where it was found that dissipation of the electromagnetic energy by magnetic reconnection can account for both the bulk acceleration of the flow and for the prompt emission.

The dynamics of fireballs and of outflows in the AC model are distinctively different. Fireballs are driven by radiation pressure and exhibit a period of rapid acceleration in which the Lorentz factor $\Gamma \propto r$, where $r$ denotes the distance from the central engine (Paczyński 1986). The flow saturates either when there is no more energy available to further accelerate the baryons or when radiation and matter decouple at the Thomson photosphere. An analysis of the dynamics of neutron-rich fireballs was recently presented by Rossi et al. (2006). The dynamics of neutrons in MHD flows was considered previously by Vlahakis et al. (2003) in the context of a different model for the outflow (Vlahakis \& Königl 2003) than the AC model considered here. In the AC model, the acceleration of the flow is quite gradual and can be approximated with $\Gamma \propto r^{1 / 3}$ (Drenkhahn 2002). Since acceleration of the flow is driven by magnetic forces, the flow can saturate far beyond the photosphere. It is expected that the difference in dynamics affects the number and the energy of secondary particles created in $n p$ collisions. Furthermore, the presence of a strong magnetic field can affect the interaction of secondary particles with the flow.

Motivated by the fact that neutrinos and $\gamma$-rays from inelastic $n p$ collisions could provide an indication about the nature of GRB outflows, we consider in this paper both fireballs and AC flows with a substantial neutron component. We investigate the dynamics of these flows and the creation of $\gamma$-rays and neutrinos in inelastic $n p$ collisions. In order to give an accurate comparison between the fireball model and the AC model, we consider both models here. Furthermore we use accurate fitting formulae for both the total and inelastic $n p$ cross sections, which has an important effect on the calculated fluences of secondary particles.

This paper is organized as follows. In Sect. 2 we discuss the dynamical behavior of fireballs and of GRB outflows described by the AC model. In Sect. 3 we consider particle creation in inelastic $n p$ collisions. We discuss here the parameter space in which the mechanism is operational and we compute the fluences and energies of secondary neutrinos and $\gamma$-rays. Detection prospects are discussed in Sect. 4 and conclusions are presented in Sect. 5.

\section{Dynamics of neutron-rich GRB flows}

Deep in the flow neutrons are strongly coupled to protons through elastic collisions, so that the two fluids behave as a single one. This $n p$ fluid is accelerated by conversion of thermal energy into kinetic energy in the fireball model and of magnetic energy into kinetic energy in the reconnection model. When the dynamical time of the flow becomes shorter than the $n p$ collision time, the two fluids decouple and the neutrons enter the coasting phase. Provided that the flow has not already reached its terminal bulk Lorentz factor, the protons keep accelerating above the $n p$ decoupling radius, which results in relative motion of the two fluids.

The analysis of the effect of a neutron component on the dynamics is made separately for the fireball and the reconnection model for the various stages of their evolution. Since the treatment of the mass flux is identical in both models, it is presented first.

\subsection{Mass flux: protons and neutrons}

For an ultrarelativistic, steady, radial flow, assumed by both models under consideration, conservation of mass implies that the baryon outflow rate obeys

$\dot{M}=\dot{M}_{\mathrm{p}}+\dot{M}_{\mathrm{n}}=4 \pi r^{2} m c\left(\Gamma_{\mathrm{p}} n_{\mathrm{p}}^{\prime}+\Gamma_{\mathrm{n}} n_{\mathrm{n}}^{\prime}\right)=4 \pi r^{2} m c \Gamma_{\mathrm{p}} n_{\mathrm{p}}^{\prime}(1+\xi)$

where $\Gamma_{\mathrm{p}}$ and $\Gamma_{\mathrm{n}}$ stand for the bulk Lorentz factor of the protons and the neutrons, respectively, and $n_{\mathrm{p}}^{\prime}$ and $n_{\mathrm{n}}^{\prime}$ for their proper number densities. The masses of protons and neutrons are assumed equal $m_{\mathrm{p}} \simeq m_{\mathrm{n}}=m$ and $\xi$ stands for the neutron-to-proton mass flux ratio:

$\xi \equiv \frac{\dot{M}_{\mathrm{n}}}{\dot{M}_{\mathrm{p}}}=\frac{\Gamma_{\mathrm{n}} n_{\mathrm{n}}^{\prime}}{\Gamma_{\mathrm{p}} n_{\mathrm{p}}^{\prime}}$

The ratio $\xi$ depends on the radius $r$ since free neutrons decay into protons on a comoving timescale $\tau_{\beta} \sim 900 \mathrm{~s}$ resulting in

$\frac{\mathrm{d} \dot{M}_{\mathrm{n}}}{\mathrm{d} r}=\frac{\mathrm{d} \dot{M}_{\mathrm{n}}}{\Gamma_{\mathrm{n}} c \mathrm{~d} t^{\prime}}=-\frac{\dot{M}_{\mathrm{n}}}{\Gamma_{\mathrm{n}} c \tau_{\beta}}$

where $t^{\prime}$ stands for the comoving time. Taking into account that a proton is produced for every neutron that decays (i.e. $\mathrm{d} \dot{M}_{\mathrm{n}} / \mathrm{d} r=-\mathrm{d} \dot{M}_{\mathrm{p}} / \mathrm{d} r$ ), Eqs. (2) and (3) yield an expression for $\xi$ as a function of radius:

$\frac{\mathrm{d} \xi}{\mathrm{d} r}=-\frac{\xi(1+\xi)}{\Gamma_{\mathrm{n}} c \tau_{\beta}}$

From Eq. (1) one can solve for the number density of protons and neutrons as a function of radius to find that

$n_{\mathrm{p}}^{\prime}=\frac{1}{1+\xi} \frac{\dot{M}}{4 \pi r^{2} m c \Gamma_{\mathrm{p}}}$,

and

$n_{\mathrm{n}}^{\prime}=\frac{\xi}{1+\xi} \frac{\dot{M}}{4 \pi r^{2} m c \Gamma_{\mathrm{n}}}$

The number density of the protons and neutrons is determined once their bulk Lorentz factor as a function of radius is derived. This is the topic of the next sections. 


\subsection{The fireball}

In the fireball model most of the energy is initially stored in the form of thermal energy $e$, which is dominated by the energy density of radiation. The luminosity $L$ of the flow is the sum of kinetic and radiation flux (e.g., Rossi et al. 2006):

$L=4 \pi r^{2} c\left[\Gamma_{\mathrm{p}}^{2}\left(4 e / 3+n_{\mathrm{p}}^{\prime} m c^{2}\right)+\Gamma_{\mathrm{n}}^{2} n_{\mathrm{n}}^{\prime} m c^{2}\right]$.

This expression can be rewritten as

$L=4 \pi r^{2} \Gamma_{\mathrm{p}}^{2} c n_{\mathrm{p}}^{\prime} m c^{2}\left(1+\xi \frac{\Gamma_{\mathrm{n}}}{\Gamma_{\mathrm{p}}}+x\right)$,

where we have defined $x \equiv 4 e / 3 n_{\mathrm{p}}^{\prime} m c^{2}$.

An important quantity for the evolution of the flow is the baryon loading parameter $\eta \equiv L / \dot{M} c^{2} \gg 1$ where $\dot{M}$ (defined in Eq. (1)) includes both the contribution of the proton and the neutron fluid. Using expressions (1) and (8) one derives the expression

$(1+\xi) \eta=\Gamma_{\mathrm{p}}(1+x)+\xi \Gamma_{\mathrm{n}}$.

Assuming that the flow starts from rest (i.e., $\Gamma_{\mathrm{p}, 0}=\Gamma_{\mathrm{n}, 0}=1$ ) at an initial radius $r_{0}$ and initial neutron-to-proton ratio $\xi_{0}$, the initial value for $x$ is $x_{0}=4 e_{0} / 3 n_{\mathrm{p}, 0}^{\prime} m c^{2}=\left(1+\xi_{0}\right)(\eta-1)$.

As long as the flow is Thomson thick, radiation and particles remain coupled and the evolution of the fireball is fully determined by the adiabatic law ${ }^{1}$

$e=e_{0}\left(\frac{n_{\mathrm{p}}^{\prime}}{n_{\mathrm{p}, 0}^{\prime}}\right)^{4 / 3}$.

From Eqs. (5), (9) and (10) one finds for the internal energy-toproton rest mass ratio in the flow

$x=x_{0}\left(\frac{n_{\mathrm{p}}^{\prime}}{n_{\mathrm{p}, 0}^{\prime}}\right)^{1 / 3}=\left(1+\xi_{0}\right)(\eta-1)\left(\frac{r_{0}^{2}}{r^{2} \Gamma_{\mathrm{p}}}\right)^{1 / 3}$.

Differentiating Eq. (9) with respect to radius $r$ and using Eq. (11), one has an expression relating the bulk Lorentz factor of the proton and the neutron fluids in the optically thick part of the flow (see also Rossi et al. 2006)

$\frac{\mathrm{d} \Gamma_{\mathrm{p}}}{\mathrm{d} r}=\frac{\Gamma_{\mathrm{p}}}{r} \frac{2 x}{2 x+3}-\frac{3 \xi_{0}}{2 x+3} \frac{\mathrm{d} \Gamma_{\mathrm{n}}}{\mathrm{d} r}$.

For the dynamics of the neutron-rich fireball to be fully determined, one needs to look closer at the momentum exchange between the neutron and the proton fluids because of $n p$ collisions. This has been studied by Derishev et al. (1999a) and Rossi et al. (2006) who showed that when the two fluids have a relative velocity $\beta_{\text {rel }}$, there is a drag force that accelerates the neutrons

$\frac{\mathrm{d} \Gamma_{\mathrm{n}}}{\mathrm{d} r}=\frac{n_{\mathrm{p}}^{\prime} \sigma_{\mathrm{tot}}}{2} \Gamma_{\mathrm{rel}}^{2} \beta_{\mathrm{rel}}^{2}$,

where $\Gamma_{\text {rel }} \simeq\left(\Gamma_{\mathrm{n}} / \Gamma_{\mathrm{p}}+\Gamma_{\mathrm{p}} / \Gamma_{\mathrm{n}}\right) / 2$ for ultrarelativistic flows and the total $n p$ scattering cross section $\sigma_{\text {tot }}$ is a function of $\beta_{\text {rel }}$. This expression accounts for the $n p$ interaction and does not depend

1 This expression does not take into account the increase of the proton density due to neutron decay. The use of this expression is justified because, for the parameter space relevant for GRB flows, there is only a negligible fraction of neutrons that decays below the photosphere of fireballs. Hereafter, in the Thomson thick part of the flow, we set $\xi=\xi_{0}$. on the acceleration mechanism (thermal or magnetic) of the flow. It can, thus, be applied to both fireballs and MHD flows.

The $n p$ scattering cross section depends on the relative velocity of the two fluids. For $n p$ scatterings that take place with energies below the pion creation threshold, the scattering cross section can with good accuracy be taken to scale as $\propto 1 /\left(c_{1} \beta_{\text {rel }}+\right.$ $c_{2} \beta_{\text {rel }}^{3}$ ), while it remains almost constant for higher energies. The constants $c_{1}$ and $c_{2}$ are found by fitting to experimental data from Yao et al. (2006; see Appendix A)

$\sigma_{\text {tot }}=\max \left[\frac{\bar{\sigma}}{0.19 \beta_{\text {rel }}+5.2 \beta_{\text {rel }}^{3}}, \bar{\sigma}\right]$,

where $\bar{\sigma} \approx 4 \times 10^{-26} \mathrm{~cm}^{2}$. Our fitting formulae for $\sigma_{\text {tot }}$ are more accurate than the expressions used by Rossi et al. (2006), where the total $n p$ scattering cross section is substantially underestimated for $\beta_{\text {rel }} \Gamma_{\text {rel }} \lesssim 1$ (i.e. before $n p$ decoupling). This results in some differences in the dynamics close to the decoupling radius. We find that the two fluids decouple over a narrower radial range (i.e. sharper decoupling). Furthermore, the fitting formula (14) results in terminal neutron Lorentz factors that are $\sim 10 \%$ higher than those found when we use the Rossi et al. (2006) expressions for the $n p$ scattering cross section.

With Eqs. (11)-(13) one has the complete description of the dynamics of the fireball in the Thomson thick part of the flow (i.e. below the photosphere). In the optically thin part radiation and matter decouple and expression (10) is no longer applicable.

Since radiation pressure is the driving mechanism of acceleration in the fireball, one would expect no further acceleration of the flow to take place above the photosphere. On the other hand, although most of the photons do not scatter with electrons above the photosphere, the electrons (outnumbered by the photons by a factor $\sim 10^{5}$ ) are still repeatedly scattered resulting in a residual acceleration of the flow in the optically thin region. This residual acceleration is given by the expression (Beloborodov 2002; Rossi et al. 2006, appropriately modified to include the neutron fluid):

$$
\frac{\mathrm{d} \Gamma_{\mathrm{p}}}{\mathrm{d} r}+\xi \frac{\mathrm{d} \Gamma_{\mathrm{n}}}{\mathrm{d} r}=\frac{\sigma_{\mathrm{T}} L_{\mathrm{r}}}{16 \pi \Gamma_{\mathrm{p}}^{2} r^{2} m c^{3}}\left[1-\left(\frac{\Gamma_{\mathrm{p}} r_{\mathrm{ph}}}{\Gamma_{\mathrm{p}}\left(r_{\mathrm{ph}}\right) r}\right)^{4}\right]+\frac{\Gamma_{\mathrm{p}}-\Gamma_{\mathrm{n}}}{1+\xi} \frac{\mathrm{d} \xi}{\mathrm{d} r}
$$

where $\sigma_{\mathrm{T}}$ is the Thomson cross section and $L_{\mathrm{r}}=16 \pi r^{2} c \Gamma_{\mathrm{p}}^{2} e / 3$ stands for the radiative luminosity of the flow. The first term in the right hand side of the last expression accounts for the residual acceleration from radiation and the second for the effect of neutron decay on the dynamics. Using Eqs. (7) and (9), we have for the radiative luminosity of the flow:

$L_{\mathrm{r}}=L\left(1-\frac{\Gamma_{\mathrm{p}}+\xi \Gamma_{\mathrm{n}}}{\eta(1+\xi)}\right)$

The expressions (4), (13), (15) and (16) describe the dynamics of the flow in the Thomson thin regime.

\subsection{The reconnection model}

In the magnetic reconnection model the flow is considered starting from the Alfvén point $r_{\mathrm{A}}$ and is dominated by Poynting flux. The luminosity of the flow is the sum of the kinetic and Poynting flux:

$L=4 \pi r^{2} c\left[\Gamma_{\mathrm{p}}^{2}\left(4 e / 3+n_{\mathrm{p}}^{\prime} m c^{2}\right)+\Gamma_{\mathrm{n}}^{2} n_{\mathrm{n}}^{\prime} m c^{2}\right]+c(r B)^{2}$,

where $B$ is the magnetic field strength in the central engine frame, which is dominated by its toroidal component. 
A detailed investigation of the properties of a neutron-free flow is presented in Drenkhahn (2002) under the assumption of a cold flow (i.e. a flow where the term $4 e / 3$ is neglected with respect the other terms in Eq. (17)). A full numerical investigation showed that the dynamical description under the cold flow assumption is rather accurate (Drenkhahn \& Spruit 2002). Hereafter, we assume that the flow is cold. One should keep in mind, however, that though of moderate dynamical significance, the internal energy of the flow - dominated by the energy density of radiation - plays a crucial role for its photospheric emission (Giannios 2006; Giannios \& Spruit 2007). Furthermore, the cold flow assumption can overestimate the acceleration of the flow in the Thomson thin region by up to $50 \%$ in the limit that the internally dissipated energy does not stay in the flow but is efficiently radiated away (Drenkhahn \& Spruit 2002). More realistically only a fraction of the dissipated energy is radiated away and the error we make in the Thomson thin region is smaller.

Setting $e=0$ and using Eqs. (1) and (17) we have

$L=\frac{\Gamma_{\mathrm{p}}+\xi \Gamma_{\mathrm{n}}}{1+\xi} \dot{M} c^{2}+c(r B)^{2}=\frac{\Gamma_{\mathrm{p}}+\xi \Gamma_{\mathrm{n}}}{1+\xi} \dot{M} c^{2}(1+\sigma)$,

where $\sigma \equiv(1+\xi)(r B)^{2} /\left(\Gamma_{\mathrm{p}}+\xi \Gamma_{\mathrm{n}}\right) \dot{M} c$ is the magnetization parameter of the flow and stands for the Poynting-to-kinetic flux ratio. Using the last expression, the baryon loading of the flow is

$\eta \equiv \frac{L}{\dot{M} c^{2}}=\frac{\Gamma_{\mathrm{p}}+\xi \Gamma_{\mathrm{n}}}{1+\xi}(1+\sigma)$.

In the reconnection model, the flow is considered starting from the Alfvén radius with magnetization $\sigma_{0}$. In the inner part of the flow the very frequent $n p$ collisions lead to $\Gamma_{\mathrm{p}, 0} \simeq \Gamma_{\mathrm{n}, 0}=$ $\sqrt{\sigma_{0}}$. In view of Eq. (19), one has that $\eta=\sqrt{\sigma_{0}}\left(1+\sigma_{0}\right) \simeq$ $\sigma_{0}^{3 / 2}$. The initial magnetization $\sigma_{0}$ is, thus, an alternative means in parameterizing the baryon loading of a PFD flow.

The radial dependence of the magnetic field strength is given by the induction equation that is appropriately modified to take into account the magnetic field dissipation through reconnection (Drenkhahn \& Spruit 2002):

$$
\frac{\mathrm{d}(r B)}{\mathrm{d} r}=-\frac{r B}{c \tau_{\mathrm{d}}} \text {. }
$$

Here,

$\tau_{\mathrm{d}}=\frac{2 \pi \Gamma_{\mathrm{p}}^{2}}{\varepsilon \Omega} \sqrt{\frac{\sigma+1}{\sigma}}$

is the dissipation timescale of the magnetic field (in the central engine frame), $\Omega$ stands for the angular frequency of the rotator, and $\varepsilon$ parameterizes the magnetic reconnection speed $v_{\text {rec. }}$. As in most models of magnetic reconnection, $v_{\text {rec }}$ scales with the Alfvén speed $v_{\mathrm{A}}$, i.e. $v_{\text {rec }}=\varepsilon v_{\mathrm{A}}$ (see, for example, Lyubarsky 2005). A nominal value used for $\varepsilon$ is 0.1 .

By combining Eqs. (4), (17), (18) and (20) one can eliminate the magnetic field $B$ and derive an equation for the bulk Lorentz factor of the protons and the neutrons:

$\frac{\mathrm{d} \Gamma_{\mathrm{p}}}{\mathrm{d} r}+\xi \frac{\mathrm{d} \Gamma_{\mathrm{n}}}{\mathrm{d} r}=\frac{2}{c \tau_{\mathrm{d}}}\left((1+\xi) \sigma_{0}^{3 / 2}-\Gamma_{\mathrm{p}}-\xi \Gamma_{\mathrm{n}}\right)+\frac{\Gamma_{\mathrm{p}}-\Gamma_{\mathrm{n}}}{1+\xi} \frac{\mathrm{d} \xi}{\mathrm{d} r}$.

The last expression, in combination with Eqs. (4) and (13), describes the the dynamics of neutron-rich flows in the reconnection model.

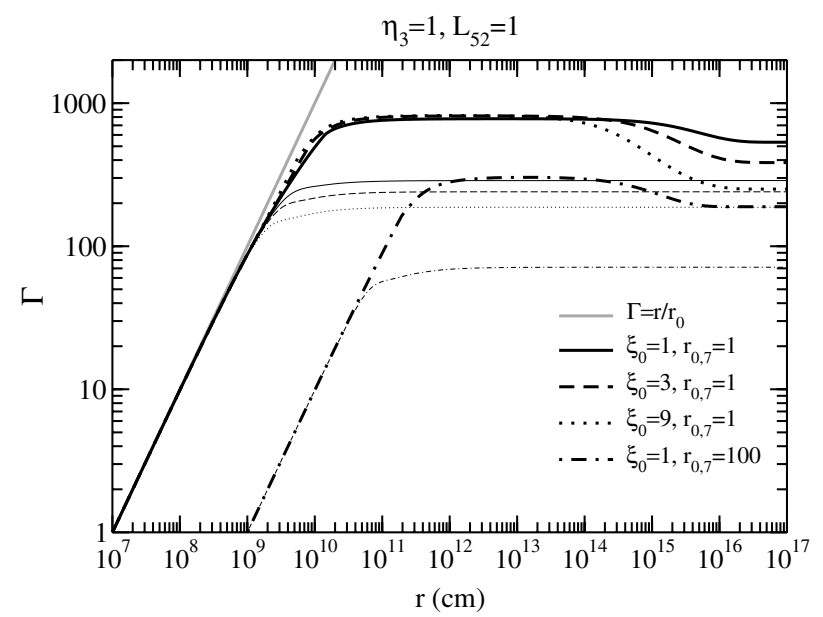

Fig. 1. Bulk Lorentz factor of the protons (thick lines) and neutrons (thin lines) for different values of the initial neutron-to-proton ratio $\xi_{0}$ and radius $r_{0}$ of the fireball. At small radii, both protons and neutrons are in the linear acceleration regime (gray line). After $n p$ decoupling the neutrons saturate while protons can be further accelerated by radiation pressure. At $r \sim 10^{15} \mathrm{~cm}$ the neutrons decay into protons that interact and decelerate the preexisting protons.

\subsection{Results}

Having derived a closed system of equations that describe the dynamics of neutron-rich flows, we proceed with the investigation of the dependence of their properties on the parameters of the flow for both fireballs and strongly magnetized flows.

\subsubsection{The fireball}

By numerically solving Eqs. (11)-(13) in the Thomson thick part of the flow and Eqs. (4), (13), (15) and (16) above the photosphere, one can follow the various stages of the neutron-rich fireball (see also Rossi et al. 2006).

In Figs. 1 and 2, the bulk Lorentz factors of the proton and the neutron fluids are plotted as function of radius for different values of the parameters of the fireball model. The latter are the luminosity of the flow $L$, the baryon loading $\eta$, the initial neutron-to-proton ratio $\xi_{0}$ and initial radius $r_{0}$ of the flow. All the models studied have $\eta \gtrsim 100$ relevant for GRB flows.

These low-baryon flows pass through an initial phase of rapid acceleration. During this phase, the neutron and proton fluids are strongly coupled and move practically with the same bulk Lorentz factor. Setting $\Gamma_{\mathrm{p}} \simeq \Gamma_{\mathrm{n}}$ in Eq. (12) we have

$\frac{\mathrm{d} \Gamma_{\mathrm{p}}}{\mathrm{d} r}=\frac{\Gamma_{\mathrm{p}}}{r} \frac{2 x}{2 x+3\left(1+\xi_{0}\right)}$.

In the limit of $x \gg 3\left(1+\xi_{0}\right) / 2$, radiation pressure leads to the well known linear acceleration of the flow as function of radius (cf. Goodman 1986; Paczyński 1986; Piran et al. 1993):

$\Gamma_{\mathrm{p}} \simeq \Gamma_{\mathrm{n}}=\frac{r}{r_{0}}$

If no $n p$ decoupling were to take place, the bulk Lorentz factor of the flow would saturate at $\Gamma_{\infty}=\eta$ at the saturation radius $r_{\mathrm{s}}=\eta r_{0}$.

Note that although at small radii the numerical results follow the linear scaling (24) closely, there are deviations from this scaling appearing for $\Gamma_{\mathrm{p}} \gtrsim 100$ for the models presented in Figs. 1 and 2. Since Eq. (24) is exact for a fireball with a 


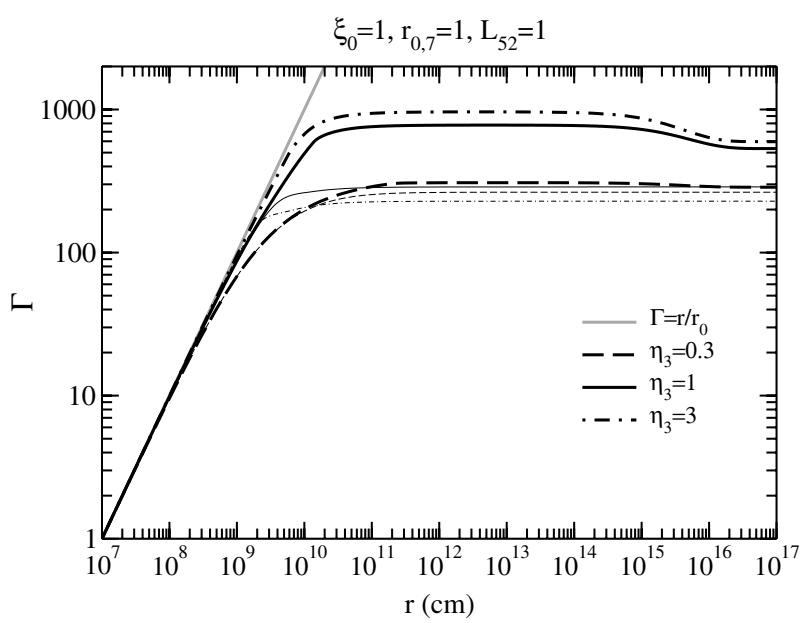

Fig. 2. Bulk Lorentz factor of the protons (thick lines) and neutrons (thin lines) for different values of the baryon loading $\eta$ of the fireball. For low baryon loading (high $\eta$ ), the protons are accelerated to much higher bulk Lorentz factors than the neutrons. For high $\eta$, the saturation of the protons takes place close to the Thomson photosphere while the photospheric emission is very powerful.

negligible number of baryons, finite- $\eta$ flows have bulk Lorentz factors $\Gamma(r)<r / r_{0}$.

At larger radii the density of the flow drops and $n p$ scatterings become less frequent. When the comoving dynamical timescale becomes shorter than the $n p$ scattering timescale, the two fluids decouple and the neutrons are not accelerated any more. The relative velocity increases rapidly at decoupling. One can define the decoupling condition as $\Gamma_{\text {rel }} \beta_{\text {rel }}=1$. Setting this condition in (13) and using also (24) one finds for the decoupling radius

$r_{\mathrm{np}}=2.6 \times 10^{9} L_{52}^{1 / 3} r_{0,7}^{2 / 3} \eta_{3}^{-1 / 3}\left(\frac{1+\xi_{0}}{2}\right)^{-1 / 3} \mathrm{~cm}$,

and for the Lorentz factor at decoupling

$\Gamma_{\mathrm{np}}=2.6 \times 10^{2} L_{52}^{1 / 3} \eta_{3}^{-1 / 3} r_{0,7}^{-1 / 3}\left(\frac{1+\xi_{0}}{2}\right)^{-1 / 3}$.

If the flow reaches its terminal Lorentz factor before $n p$ decoupling has taken place, both the neutron and proton flows coast with the same speed.

For a flow with a sufficiently high $\eta$, i.e.

$\eta>\eta_{\mathrm{cr}} \equiv 360 L_{52}^{1 / 4} r_{0,7}^{-1 / 4}\left(\frac{1+\xi_{0}}{2}\right)^{-1 / 4}$,

the protons keep being accelerated after $n p$ decoupling has taken place while the neutrons coast with $\Gamma_{\mathrm{n}} \sim \Gamma_{\mathrm{np}}$. The bulk Lorentz factor at $n p$ decoupling $\Gamma_{\mathrm{np}}$ provides a good estimate of the saturation Lorentz factor of the neutrons $\Gamma_{\mathrm{n}, \mathrm{s}}$. To quantify this statement, we have compared the analytical estimate for $\Gamma_{n p}$ with the numerical values of $\Gamma_{\mathrm{n}}$ at a large radius (here taken at $r=10^{17} \mathrm{~cm}$ ) and found that the two quantities agree with each other within $\sim 25 \%$ for the (rather large) parameter space $\eta_{\text {cr }}<\eta<3000,0.01<L_{52}<10,0<\xi_{0}<10$ and $1<r_{0,7}<100$.

When condition (27) is satisfied, the protons are further accelerated by radiation pressure after $n p$ decoupling until either all internal energy has been used or the flow crosses the photosphere, where the flow becomes transparent with respect to Thomson scattering so that radiation and matter decouple.
An estimate of the maximum Lorentz factor of the protons is given by assuming a neutron-free flow after $n p$ decoupling with luminosity $\hat{L}$ that does not include the kinetic energy of neutrons (i.e. $\left.\hat{L}=L-\Gamma_{\mathrm{np}} \xi_{0} \dot{M} c^{2} /\left(1+\xi_{0}\right)\right)$ and mass flux $\hat{\dot{M}}=\dot{M} /\left(1+\xi_{0}\right)$. The baryon loading of the decoupled proton flow is

$\hat{\eta}=\frac{\hat{L}}{\hat{\dot{M}} c^{2}}=\eta\left(1+\xi_{0}\right)-\xi_{0} \Gamma_{\text {np }}$.

The acceleration of the proton fluid will saturate at

$\Gamma_{\mathrm{p}, \mathrm{s}}=\min \left[\hat{\eta}, \hat{\eta}_{\mathrm{rad}}\right]$,

where $\hat{\eta}_{\mathrm{rad}}=\left(\hat{L} \sigma_{\mathrm{T}} / 4 \pi r_{0} m c^{3}\right)^{1 / 4}$ gives the terminal Lorentz factor of the protons when the acceleration of the flow is limited by photospheric crossing (Beloborodov 2002). This estimate takes into account the residual acceleration in the optically thin region discussed in Sect. 2.2.

At still larger radii of the order of $r_{\beta}=\Gamma_{\mathrm{np}} c \tau_{\beta} \sim$ $10^{15}-10^{16} \mathrm{~cm}$, neutron decay has an appreciable effect on the dynamics of the flow. The neutrons decay into protons and interact with the faster moving proton flow, thereby slowing it down. Note that at distances $10^{17} \mathrm{~cm}$, practically all the neutrons have decayed. The terminal Lorentz factor of the protons there is $\Gamma_{\mathrm{p}, \infty} \leq \eta$. For flows with $\hat{\eta}>\hat{\eta}_{\text {rad }}$, most of the energy is not used to accelerate the baryons (resulting in $\Gamma_{\mathrm{p}, \infty} \ll \eta$ ) but instead appears as photospheric emission of the flow.

Further out, the flow enters the afterglow phase where it decelerates because of interaction with the circumburst medium. This last phase is not considered in this study.

\subsubsection{The reconnection model}

We now present the various phases of the development of the flow in the context of the reconnection model. The neutron-free flow has been studied by Drenkhahn (2002) and Drenkhahn \& Spruit (2002). Here we focus on the dynamical effect of the neutrons. In Figs. 3 and 4, the bulk Lorentz factors of the proton and the neutron fluids are plotted as function of radius for different values of the parameters of the reconnection model. These parameters are the luminosity of the flow $L$, the initial magnetization $\sigma_{0}$ of the flow (that also parameterizes the baryon loading since $\eta \simeq \sigma_{0}^{3 / 2}$ ), the initial neutron-to-proton ratio $\xi_{0}$ and the combination $\varepsilon \Omega$ that parameterizes the reconnection speed.

The flow passes through an initial phase of acceleration where the neutron and proton fluids are strongly coupled and move practically with the same bulk Lorentz factor. Setting $\Gamma_{\mathrm{p}} \simeq \Gamma_{\mathrm{n}}$ in Eq. (22) we have

$\frac{\mathrm{d} \Gamma_{\mathrm{p}}}{\mathrm{dr}}=\frac{\varepsilon \Omega \sqrt{1-\Gamma_{\mathrm{p}} / \sigma_{0}^{3 / 2}}}{\pi c \Gamma_{\mathrm{p}}^{2}}\left(\sigma_{0}^{3 / 2}-\Gamma_{\mathrm{p}}\right)$.

In the limit of $\Gamma_{\mathrm{p}} \ll \sigma_{0}^{3 / 2}$ (i.e. the flow is still dominated by Poynting flux), the last equation can be integrated analytically to find (Drenkhahn 2002):

$\Gamma=\left(\frac{3 \varepsilon \Omega \sigma_{0}^{3 / 2}}{\pi c}\left(r-r_{0}\right)+\sigma_{0}^{3 / 2}\right)^{1 / 3}$,

The reconnection model predicts a gradual acceleration of the flow $\Gamma \sim r^{1 / 3}$ in the regime $\sqrt{\sigma_{0}} \ll \Gamma_{\mathrm{p}} \ll \sigma_{0}^{3 / 2}$ with the bulk Lorentz factor of the flow given by

$\Gamma_{\mathrm{p}} \simeq \Gamma_{\mathrm{n}}=\left(3 \varepsilon \Omega \sigma_{0}^{3 / 2} r / \pi c\right)^{1 / 3}$. 


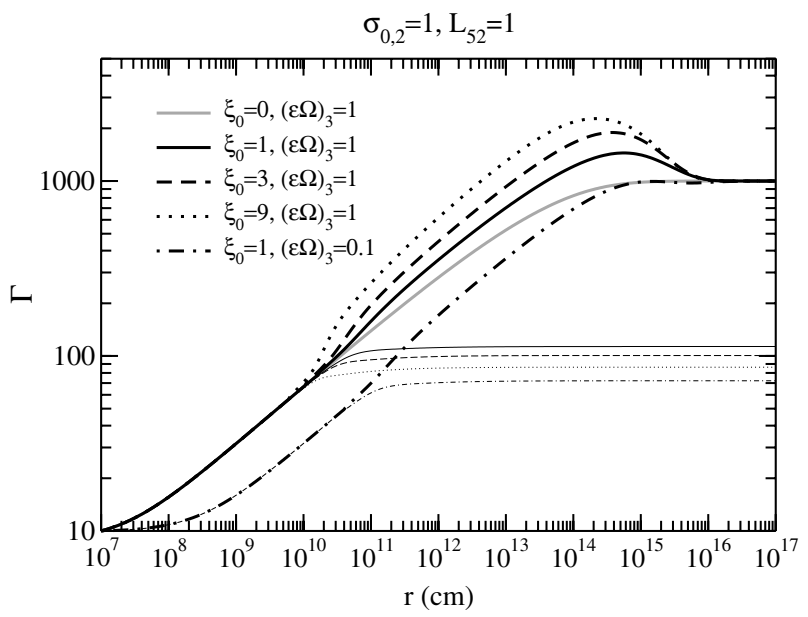

Fig. 3. Bulk Lorentz factors of the protons (thick lines) and neutrons (thin lines) for different values of the initial neutron-to-proton ratios $\xi_{0}$ and reconnection speed parameterized by $\varepsilon \Omega$ in the reconnection model. At $n p$ decoupling radius the acceleration rate of the protons is enhanced. This effect is particularly pronounced for $\xi_{0} \gg 1$ flows. At $r \sim 10^{14}-10^{15} \mathrm{~cm}$, the neutrons decay causing deceleration of the protons.

This expression is valid as long as the neutrons have not decoupled from the protons and the flow has not reached its terminal Lorentz factor $\Gamma_{\infty}=\sigma_{0}^{3 / 2}$ at the saturation radius

$r_{\mathrm{s}}=\frac{\pi c}{3 \varepsilon \Omega} \sigma_{0}^{3}$.

At larger radii the density of the flow drops and nuclear scatterings become less frequent. When the comoving dynamical timescale becomes shorter than the $n p$ scattering timescale, the two fluids decouple and the neutrons are not accelerated any more. Their relative velocity $\beta_{\text {rel }}$ increases rapidly around decoupling. As for fireballs, one can define the decoupling condition as $\Gamma_{\text {rel }} \beta_{\text {rel }}=1$. Setting this condition in Eq. (13) and using also Eq. (32) one finds for the decoupling radius

$r_{\mathrm{np}}=4.1 \times 10^{10} L_{52}^{3 / 5}(\varepsilon \Omega)_{3}^{-2 / 5} \sigma_{0,2}^{-3 / 2}\left(\frac{1+\xi_{0}}{2}\right)^{-3 / 5} \mathrm{~cm}$.

The bulk Lorentz factor of the flow at the decoupling is

$\Gamma_{\mathrm{np}}=110 L_{52}^{1 / 5}(\varepsilon \Omega)_{3}^{1 / 5}\left(\frac{1+\xi_{0}}{2}\right)^{-1 / 5}$.

If the flow reaches its terminal Lorentz factor at $r_{\mathrm{s}}$ before $n p$ decoupling has taken place, both the neutron and proton flow coast with the same speed. For a flow with a sufficiently high $\sigma_{0}$, such that

$\sigma_{0}>\sigma_{\mathrm{cr}} \equiv 23 L_{52}^{2 / 15}(\varepsilon \Omega)_{3}^{2 / 15}\left(\frac{1+\xi_{0}}{2}\right)^{-2 / 15}$,

the protons are further accelerated after $n p$ decoupling has taken place while the neutrons coast with $\Gamma_{\mathrm{n}} \sim \Gamma_{\mathrm{np}}$. The bulk Lorentz factor at $n p$ decoupling $\Gamma_{\mathrm{np}}$ provides a good estimate of the saturation Lorentz factor of the neutrons $\Gamma_{\mathrm{n}, \mathrm{s}}$. Comparing the analytical estimate for $\Gamma_{\mathrm{np}}$ with the numerical values of $\Gamma_{\mathrm{n}}$ at large radii (taken here at $r=10^{17} \mathrm{~cm}$ ), we have found that the two quantities agree with each other within $\sim 10 \%$ for the parameter space $\sigma_{\mathrm{cr}}<\sigma_{0}<300,0.01<L_{52}<10,0<\xi_{0}<10$ and $0.01<(\varepsilon \Omega)_{3}<10$.

The critical value $\sigma_{\mathrm{cr}}$ corresponds to baryon loading $\eta_{\mathrm{cr}} \simeq$ $\sigma_{0, c r}^{3 / 2} \sim 100$. For baryon loadings $\eta \gtrsim 100$ relevant for GRB

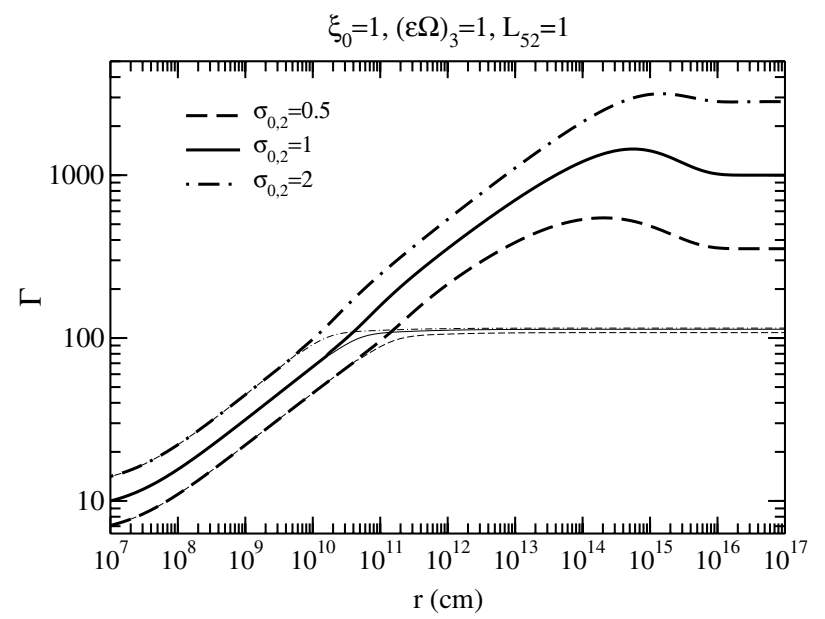

Fig. 4. Bulk Lorentz factors of the protons (thick lines) and neutrons (thin lines) for different baryon loadings parameterized by the magnetization parameter $\sigma_{0}$ in the reconnection model. The bulk Lorentz factor of the neutrons at $n p$ decoupling is essentially independent of $\sigma_{0}$, in agreement with the analytical estimate (35).

flows, $n p$ decoupling takes place before the saturation radius has been crossed. In this case a substantial amount of magnetic energy is dissipated at radii $r>r_{\mathrm{np}}$, which is used to accelerate the protons.

At the $n p$ decoupling radius the flow becomes effectively less baryon loaded and the protons increase their Lorentz factor more rapidly than the $\Gamma_{\mathrm{p}} \sim r^{1 / 3}$ scaling. This enhanced acceleration is particularly pronounced in neutron dominated flows (where $\xi_{0} \gg 1$; see Fig. 3). A similar enhancement in the acceleration has been found by Vlahakis et al. (2003) in the context of a different MHD model for GRBs.

Note that soon after $n p$ decoupling has taken place the flow crosses the Thomson photosphere. The protons keep accelerating after the photospheric crossing in the magnetized flow since the acceleration is magnetic and not driven by radiation pressure as in the fireball model. At larger radii, the protons can reach bulk Lorentz factors in excess of the limit $\sigma_{0}^{3 / 2}$ that characterizes a pure proton flow (shown with dotted line in Fig. 3).

At larger radii the neutrons undergo beta decay. For high- $\xi_{0}$ flows, at radius $r \sim \Gamma_{\mathrm{np}} c \tau_{\beta} / \xi_{0}$ the number of neutrons that have decayed is comparable with the initial number of protons in the flow and the effect of neutron decay on the bulk motion of the protons becomes appreciable.

After magnetic dissipation has ceased and most of the neutrons have decayed, all the available energy has been transferred to the protons. The bulk Lorentz factor of the protons at large radii saturates to the value $\Gamma_{\mathrm{p}, \infty}=\sigma_{0}^{3 / 2}$. This takes place at $r \sim 10^{16} \mathrm{~cm}$. At these radii the flow is expected to enter the afterglow phase which is not considered here.

\section{Particle creation in inelastic neutron-proton collisions}

In the previous section we demonstrated that for low enough baryon loading, the neutrons decouple before the acceleration of the flow is completed in both fireballs and PFD flows. This leads to neutrons and protons developing relative motions and to energetic $n p$ collisions. Here, we study the production of pions through inelastic $n p$ collisions in the relativistic outflow and the subsequent decay of pions into $\gamma$-rays and neutrinos. We present 
analytical estimates for the secondary particle fluences and energies, and compare these estimates with numerical results based on the model discussed in Sect. 2.

For the analytical estimates, we approximate the proton and neutron Lorentz factors as follows:

$\Gamma_{\mathrm{p}} \simeq\left(\frac{r}{r_{0}}\right)^{p} ; \quad \Gamma_{\mathrm{n}} \simeq \min \left[\Gamma_{\mathrm{p}}, \Gamma_{\mathrm{np}}\right]$,

where $\Gamma_{\mathrm{np}}$ is the Lorentz factor of the flow at decoupling, $p$ is a model parameter that allows us to consider the fireball model and the reconnection model together ( $p=1$ for fireballs and $p=1 / 3$ for the reconnection model), and $r_{0}$ is a suitable length scale. For the fireball model $r_{0}$ is the initial radius where the fireball in injected, which is a free parameter of the model. In the reconnection model $r_{0} \equiv \pi c / 3 \varepsilon \Omega \sigma_{0}^{3 / 2}$ is a length scale defined by the specific combination of the parameters - it has no deeper physical meaning but merely serves in rewriting the expression (32) in a more compact form.

Using the unifying notation (37) for the bulk Lorentz factor of the protons and the neutrons, we express the $n p$ decoupling radius and the Lorentz factor at decoupling as:

$r_{\mathrm{np}}=\left(\frac{\bar{\sigma} L r_{0}^{2 p}}{8 \pi p m c^{3}(1+\xi) \eta}\right)^{\frac{1}{2 p+1}} ;$

$\Gamma_{\mathrm{np}}=\left(\frac{\bar{\sigma} L}{8 \pi r_{0} p m c^{3}(1+\xi) \eta}\right)^{\frac{p}{2 p+1}}$,

which combines Eqs. (25), (26), (34) and (35).

\subsection{The pion production radius}

For sufficiently low baryon loading in the flow, pion creation in inelastic $n p$ collisions is possible after $n p$ decoupling and the subsequent acceleration of the protons with respect to the neutrons. We define the pion creation radius $r_{\pi}$ as the minimum radius where the relative velocity between decoupled neutrons and protons is large enough to create pions through inelastic $n p$ collisions.

The production of a secondary particle with mass $\mu$ requires center-of-mass energy $\sqrt{s}>2 m c^{2}+\mu c^{2}$. Assuming that $\Gamma_{\mathrm{p}}(r) \gg$ 1 and $\Gamma_{\mathrm{n}}(r) \gg 1$ at radii $r>r_{\mathrm{np}}$, and taking the $n p$ collision angle equal to zero (tail-on collisions), we express the center-of-mass energy $\sqrt{s}$ as

$\sqrt{s}=m c^{2}\left(\chi^{1 / 2}+\chi^{-1 / 2}\right)$,

where we introduce the useful quantity

$\chi(r) \equiv \frac{\Gamma_{\mathrm{p}}(r)}{\Gamma_{\mathrm{n}}(r)}$.

From Eq. (40), we find that pions (which are the lightest mesons) can only be created if $\chi(r)>\chi_{\pi}$, where $\chi_{\pi^{0}}=2.13$ corresponds to neutral pion production and $\chi_{\pi^{ \pm}}=2.16$ to charged pion production. We will use the average value $\chi_{\pi}=2.15$ in this work. Using the approximate proton and neutron Lorentz factors expressed in Eqs. (37), we find that

$r_{\pi} \simeq \chi_{\pi}^{1 / p} r_{\mathrm{np}}$,

where the decoupling radius $r_{\mathrm{np}}$ is given in Eq. (38). The radius from which pions can be created is thus substantially larger than the decoupling radius. Since the density of the flow and hence the number of $n p$ scatterings decrease rather steeply with radius, it is important to discriminate between $r_{\mathrm{np}}$ and $r_{\pi}$ when considering particle creation in inelastic $n p$ scatterings.

Pion creation by $n p$ interactions occurs only when the pion creation radius $r_{\pi}$ is reached before the flow saturates. For the fireball model, saturation of the bulk Lorentz factor of the flow occurs either when there is no more energy available to further accelerate the baryons or when the flow crosses the photosphere (cf. Eq. (29)). It can be shown that saturation occurs beyond the pion creation radius only if the baryon loading of the flow is sufficiently small. We express this condition as $\eta>\eta_{\pi}$, where $\eta_{\pi}$ is the critical value for inelastic $n p$ collisions to occur in the flow. Approximating the proton and neutron Lorentz factors with Eqs. (37), we estimate that

$\eta_{\pi} \simeq 5.1 \times 10^{2} L_{52}^{1 / 4} r_{0,7}^{-1 / 4} \psi\left(\xi_{0}\right)$,

where

$\psi\left(\xi_{0}\right) \equiv 0.85\left(\chi_{\pi}+\xi_{0}\right)^{3 / 4}\left(1+\xi_{0}\right)^{-1}$

is a slowly-varying function normalized so that $\psi(1)=1$. In deriving Eq. (43) we take neutron decoupling into account by using the neutron-free luminosity $\hat{L}$ and mass flux $\hat{\dot{M}}$ as defined in Sect. 2.4.1. The numerical investigation of the fireball dynamics (see Sect. 2.4.1) shows that the proton Lorentz factors are substantially below the $\Gamma_{\mathrm{p}} \propto r$ scaling solution around $n p$ decoupling. As a result, the proton-neutron relative velocity is smaller and the pion production radius is pushed outward with respect to the analytical estimate (42). This effect makes it more difficult to create pions in the flow and requires $\eta$ to be higher than the estimate (43). Using the numerical model discussed in Sect. 2 we find that inelastic $n p$ collisions in fireballs occur generally when $\eta / \eta_{\pi} \gtrsim 2$, where $\eta_{\pi}$ is expressed in Eq. (43). For neutronrich flows $\left(\xi_{0} \gtrsim 3\right)$, neutron decoupling results in a relatively pure flow so that the protons follow the scaling approximation (43) more closely and inelastic $n p$ collisions occur already when $\eta / \eta_{\pi} \gtrsim 1.5$. Nevertheless, these results place quite stringent conditions on the fireball model parameters so that only a small fraction of GRB fireballs is expected to exhibit inelastic $n p$ collisions between bulk protons and neutrons.

For the AC model we find that, similar to the fireball case, inelastic $n p$ collisions only occur for a sufficiently low baryon loading. We express this as $\sigma_{0}>\sigma_{0, \pi}$, where we use Eqs. (37) to estimate that

$\sigma_{0, \pi} \simeq 38 \times L_{52}^{2 / 15}(\epsilon \Omega)_{3}^{2 / 15}\left(\frac{2}{1+\xi_{0}}\right)^{2 / 15}$.

We find that Eq. (45) is consistent with the critical value for $\sigma_{0}$ obtained from numerical results on the proton and neutron dynamics (using the numerical model described in Sect. 2). This value of $\sigma_{0, \pi}$ corresponds to a critical baryon loading for inelastic $n p$ collisions $\eta_{\pi}=\sigma_{0, \pi}^{3 / 2} \sim 230$ which is much lower than the critical value required in fireballs. Inelastic $n p$ collisions thus take place for a larger range of the parameter space in the reconnection model with respect to the fireball.

The strength of any neutrino and $\gamma$-ray emission that is a result of the decay of the products (mainly pions) of these collisions depends critically on the optical depth to inelastic $n p$ scattering. The calculation of this optical depth is the topic of the next section.

\subsection{Optical depth for inelastic np collisions}

The optical depth $\mathrm{d} \tau$ for a neutron with velocity $c \beta_{\mathrm{n}}$ to scatter inelastically with a population of protons with velocity $c \beta_{\mathrm{p}}$ and 
proper density $n_{\mathrm{p}}^{\prime}$ within $r \ldots r+\mathrm{d} r$ is given by (see, e.g., Landau \& Lifshitz 1971)

$\mathrm{d} \tau=\sigma_{\text {inel }} \Gamma_{\mathrm{p}} n_{\mathrm{p}}^{\prime}\left(\frac{\beta_{\mathrm{p}}-\beta_{\mathrm{n}}}{\beta_{\mathrm{n}}}\right) \mathrm{d} r \simeq \frac{\sigma_{\text {inel }} n_{\mathrm{p}}^{\prime}}{2 \Gamma_{\mathrm{n}}}\left(\chi-\frac{1}{\chi}\right) \mathrm{d} r$,

where we assume in the last approximation that $\Gamma_{\mathrm{p}} \gg 1$ and $\Gamma_{\mathrm{n}} \gg 1$ and that the collisions are tail-on.

At low center-of-mass energies the elastic and inelastic $n p$ cross sections are energy dependent. We find that for $\chi_{\pi} \leq \chi \lesssim$ 10 (which is the range of interest here) the elastic cross section is well described with $\sigma_{\mathrm{el}}(\chi)=0.75 \bar{\sigma} / \ln \chi$, where $\bar{\sigma} \equiv$ $4 \times 10^{-26} \mathrm{~cm}^{2}$. A comparison between this approximation and experimental data on the elastic cross section taken from Yao et al. (2006) is presented in appendix A. In the following, we express the inelastic $n p$ cross section as

$\sigma_{\text {inel }}\left(\chi>\chi_{\pi}\right)=\bar{\sigma}\left(1-\frac{0.75}{\ln \chi}\right)$.

We note here that the energy dependence of the $n p$ inelastic cross section has an important effect on the optical depth. If one assumes a constant cross section $\sigma_{\text {inel }}=3 \times 10^{-26} \mathrm{~cm}^{2}$ (as is often done in the literature) the optical depths are larger by a factor $\sim 4$ for both the fireball model and the reconnection model. Hence, the more realistic cross section adopted in this work leads to substantially lower estimates for the number of created particles.

We consider, in general, the situation that neutrons coast with a constant Lorentz factor $\Gamma_{\mathrm{n}}$ while protons are accelerated up to infinity with a Lorentz factor $\Gamma_{\mathrm{p}} \propto r^{p}$. Keeping $p$ as a free parameter, we integrate Eq. (46) through the flow to find that

$\tau(p)=\int_{\chi_{\pi}}^{\infty} \mathrm{d} \chi\left(1-\frac{0.75}{\ln \chi}\right)\left(\chi^{-1}-\chi^{-3}\right) \chi^{-1 / p}$,

where Eq. (38) was used to eliminate all parameters but $p$. We thus find that the optical depth for inelastic $n p$ scattering is independent of any model parameters but the dynamical power-law index $p$. This result is valid for all outflows with $\Gamma_{\mathrm{p}} \propto r^{p}$ and $\Gamma_{\mathrm{n}}=$ const, provided that $r_{\mathrm{s}} \gg r_{\pi}$. When $r_{\mathrm{s}} \gtrsim r_{\pi}$, such as in the fireball model, it represents an upper limit.

For fireballs ( $p=1)$ we find from Eq. (48) that $\tau^{\mathrm{FB}}<0.2$, which is an upper limit because the flow saturates close to the decoupling radius. The situation is complicated by the fact that saturation of the fireball can be due to energy requirements or due to crossing of the photosphere. The numerical results presented in Sect. 2.4.1 indicate that both effects cause the flow to accelerate considerably more slowly than the scaling approximation $\Gamma_{\mathrm{p}} \propto r$ near the pion creation radius $r_{\pi}$. This pushes the pion creation radius outward and decreases the optical depth for inelastic $n p$ scattering. We compute the optical depth numerically by a straightforward numerical integration of Eq. (46) using the values of $\Gamma_{\mathrm{p}}(r)$ and $\Gamma_{\mathrm{n}}(r)$ obtained with the numerical model ${ }^{2}$ discussed in Sect. 2. We find that for the parameter space $3.5 \eta_{\pi}<\eta<5000,0.01<L_{52}<10,0.3<\xi<10$, and $1<r_{0,7}<100$ the optical depth is approximated to within $\sim 25 \%$ by

$\tau^{\mathrm{FB}} \simeq 0.11\left(1-\frac{2 \eta_{\pi}}{\eta}\right)$

${ }^{2}$ In the numerical analysis, we use a more accurate but also more elaborate approximation (see Appendix A) for the cross section than the one given in Eq. (47), which results in lower optical depths. Because dynamical effects, discussed in the text, have a larger influence on the optical depth we use expression (47) for simplicity to derive an analytical estimate.
In the (rather favorable for frequent inelastic scatterings) case where $\eta=5000$ and $\xi=5$, the optical depth is $\tau^{\mathrm{FB}} \simeq 0.1$. For lower values of the baryon-loading parameter $\left(\eta / \eta_{\pi}<3.5\right)$ the optical depth is smaller than the value given in Eq. (49). A representative value for a fireball with $\eta$ a few times the critical value $\eta_{\pi}$ is $\tau^{\mathrm{FB}} \simeq 0.05$.

For the reconnection model the saturation radius $r_{\mathrm{s}}$ is typically much larger than $r_{\pi}$. We can therefore estimate the optical depth $\tau^{\mathrm{AC}}$ for an inelastic $n p$ interaction assuming that the protons are accelerated to infinity. (In principle this overestimates the interaction probability, but the difference is very small because the interaction probability decreases rapidly with $r$.) Inserting $p=1 / 3$ in Eq. (48), we find that $\tau^{\mathrm{AC}} \simeq 8 \times 10^{-3}$. This value is consistent with numerical results for flows with $\xi_{0} \sim 1$. For reference values of the parameters $L_{52}=\xi_{0}=\sigma_{0,2}=$ $(\epsilon \Omega)_{3}=1$, we find also numerically that $\tau^{\mathrm{AC}}=8 \times 10^{-3}$. For high values of $\xi_{0}$ (neutron-rich flows), the extra acceleration of the flow after neutron decoupling (discussed in Sect. 2.4.2) increases the optical depth by a factor few. We find that for the parameter space $1.5<\sigma_{0} / \sigma_{0, \pi}<10,0.01<L_{52}<100,0.3<\xi_{0}<10$, and $0.01<(\epsilon \Omega)_{3}<10$ the optical depth is approximated to within $\sim 25 \%$ by

$\tau^{\mathrm{AC}} \simeq 0.01 \xi_{0}^{1 / 2}$

In particular, the optical depth increases to $\tau^{\mathrm{AC}} \simeq 0.03$ for very neutron-rich flows $\left(\xi_{0} \simeq 10\right)$. For $1<\sigma_{0} / \sigma_{0, \pi}<1.5$ pion creation is marginally possible and the optical depth is smaller than the value obtained by Eq. (50). For very pure flows $\left(\sigma_{0} / \sigma_{0, \pi} \gtrsim 10\right)$ neutrons decouple very early (before power-law acceleration $\Gamma \propto r^{p}$ is reached), which results in an optical depth smaller by a factor $\sim 2$ than the estimate given in Eq. (50).

The obtained optical depth for inelastic $n p$ collisions is the first step in calculating the fluences of secondary pions and their decay products. The calculation of the fluences and energies of stable decay products requires a model for the average number and average energy of neutrinos and $\gamma$-rays created by $n p$ interactions. In the following sections we consider in detail the production of pions and the subsequent decay into neutrinos and $\gamma$-rays.

\subsection{Pion production}

For the collisions studied in this work, the typical incident energy of the proton measured in the rest frame of the neutron is $p_{\mathrm{p}}^{\prime} \sim 1 \mathrm{GeV} / \mathrm{c}$. In this regime experimental data on pion creation in $n p$ collisions is scarce and there is no unambiguous theoretical framework. The available data (in particular, Prokoshin \& Tiapkin 1957; Kleinschmidt et al. 1980; Daum et al. 2002; see also electronic data files available at the PPDS website http://wwwppds. ihep.su: 8001/) show a rapid rise in the single-pion cross sections just above threshold, and indicate that two-pion exclusive production cross sections are comparable to one-pion exclusive cross sections for incident proton energies (as observed in the neutron rest frame $K^{\prime}$ ) $p_{\mathrm{p}}^{\prime} \sim 2 \mathrm{GeV} / c$. Furthermore, the ratio of $\pi^{0}: \pi^{ \pm}$depends on energy; the ratio $\pi^{-}: \pi^{+}$is $1: 1$ under the assumption of nuclear isospin symmetry.

Based on the available data for incident proton energies $p_{\mathrm{p}}^{\prime} \sim$ $1 \mathrm{GeV} / c$ we model the energy distribution and average number of pions resulting from a $n p$ collisions as follows. We estimate the ratio of created pions as $\pi^{0}: \pi^{+}: \pi^{-}=2: 1: 1$. Hence the average 
number of neutrinos ${ }^{3}$ and $\gamma$-rays resulting from a single inelastic $n p$ collision is:

$\mathcal{N}_{\gamma}=1.0 ; \quad \mathcal{N}_{v_{\mu}}=1.0 ; \quad \mathcal{N}_{v_{\mathrm{e}}}=0.5$.

Experimental data indicate that, for incident proton energies $p_{\mathrm{p}}^{\prime}=1.14 \mathrm{GeV} / c$, the distribution of kinetic energy $T \equiv$ $E-m_{\pi} c^{2}$ for $\pi^{+}$mesons peaks around $0.6 T_{\max }$, where $T_{\max }$ is the maximum kinetic energy that can be carried by the pion (Kleinschmidt et al. 1980). For $\pi^{0}$ mesons in the center-of-mass $(\mathrm{COM})$ frame this ratio is almost unity below $p_{\mathrm{p}}^{\prime}<1.06 \mathrm{GeV} / c$ and decreases to $T_{\text {peak }}=0.5 T_{\max }$ at $p_{\mathrm{p}}^{\prime}=1.29 \mathrm{GeV} / c$ (Prokoshin \& Tiapkin 1957). Here we assume a constant fraction of 0.6 for all pion species and take the average pion energy for a single $n p$ collision in the COM frame $K^{\prime \prime}$ equal to the peak energy:

$\left\langle\epsilon_{\pi}^{\prime \prime}\right\rangle=\epsilon_{\pi, \text { peak }}^{\prime \prime}=0.6 \epsilon_{\pi, \text { max }}^{\prime \prime}+0.4 m_{\pi} c^{2}$,

where the maximum pion energy is equal to

$\epsilon_{\pi, \max }^{\prime \prime}=\frac{s-4 m^{2} c^{4}+m_{\pi}^{2} c^{4}}{2 \sqrt{s}}$,

and Eq. (40) relates the center-of-mass energy $\sqrt{s}$ to $\chi$. In these equations, the parameter $\chi$ provides the only reference to where the collision has occurred in the developing flow.

When the angular distribution of pions in the COM frame is known, one can derive the full particle distribution of the decay products and transform this to the observer frame in order to find the secondary energy as observed on earth. However, there is to our knowledge no accurate parameterization of the angular distribution of secondary pions created in $n p$ collisions. In the absence of such a parameterization we estimate the average observed energy of neutrinos by boosting to the observer frame from an intermediate frame in which the secondary particles are assumed to be isotropic ${ }^{4}$.

Pions are created approximately isotropically in the COM frame of the $n p$ collision. When neither pions nor their decay products are affected by the flow, as is the case for neutrino production in the fireball model, the distribution of the daughter particles can be taken to be isotropic in the COM frame. In the AC model however, the strong magnetic field deflects the charged pions significantly since the pion gyration period is much shorter than the pion decay time. We assume that in this case the pions will be distributed isotropically in the frame comoving with the proton fluid. (Any randomized component of the magnetic field will further contribute to isotropization in this frame.) Furthermore, in both the fireball model and the AC model $\gamma$-rays from neutral pion decay will interact with the soft photon field of the flow, resulting in the emission of lowerenergy photons. In the following sections these issues are discussed and estimates are presented for typical neutrino and $\gamma$-ray energies.

The decay of a charged pion also yields one electron or positron with energy $\sim 35 \mathrm{MeV}$. These contribute to the $\gamma$-ray emission which is discussed in Sect. 3.5.

\footnotetext{
${ }^{3}$ Here and in the following $v_{\mu}$ denotes both muon-neutrinos and -antineutrinos (and similar for electron-neutrinos).

${ }^{4}$ We note that, in the literature, there are various choices regarding the frame (e.g. the neutron rest frame or the proton rest frame) in which the energy distribution of $\gamma$-rays and neutrinos is computed before applying the final boost to the observer frame. Any intermediate frame leads to the same results in the observer frame provided that the angular structure of the particle distributions is taken into account. If an isotropic distribution is assumed, the choice of intermediate frame is important and depends on the physics.
}

\subsection{Observed neutrino energy}

In the fireball model pions do not interact significantly with the flow so that neutrinos from charged pion decay can be taken to be distributed isotropically in the COM frame $K^{\prime \prime}$ of the $n p$ collision. For a given value of $\chi$, the average energy of the produced neutrinos in this frame is then

$\left\langle\epsilon_{v}^{\prime \prime}\right\rangle=\frac{\left\langle\epsilon_{\pi}^{\prime \prime}\right\rangle \epsilon_{v}^{0}}{m_{\pi} c^{2}}$

where $\epsilon_{v}^{0} \simeq 35 \mathrm{MeV}$ denotes the average neutrino energy in the rest frame of the decaying pion. The average observed energy $\left\langle\epsilon_{v}\right\rangle$ is obtained by boosting to the observer frame with Lorentz boost factor $\Gamma_{\mathrm{COM}}=\sqrt{\Gamma_{\mathrm{p}} \Gamma_{\mathrm{n}}}$ (Appendix B contains a summary of frames and Lorentz factors used in this work):

$\left\langle\epsilon_{v}^{\mathrm{FB}}\right\rangle=\frac{\Gamma_{\mathrm{COM}}}{1+z} \frac{\left\langle\epsilon_{\pi}^{\prime \prime}\right\rangle \epsilon_{v}^{0}}{m_{\pi} c^{2}} \simeq \frac{\Gamma_{\mathrm{np}} \chi^{1 / 2}}{1+z} \frac{\left\langle\epsilon_{\pi}^{\prime \prime}\right\rangle \epsilon_{v}^{0}}{m_{\pi} c^{2}}$,

where $z$ is the redshift of the source. In the last equality, we approximate the proton and neutron dynamics by Eqs. (37).

For fireballs the flow saturates close to the pion creation radius and the bulk of the collisions occur when $\chi$ is equal to the saturation value $\chi_{\mathrm{s}}$. For flows with large $\eta \gtrsim 700$, where saturation is reached due to crossing of the photosphere, the terminal Lorentz factor of the flow equals $\Gamma_{\mathrm{p}, \mathrm{s}}=\hat{\eta}_{\text {rad }}$ (see Eq. (29)). In this case, the critical value $\chi_{\mathrm{s}}$ can be estimated using the scaling law expressed in Eqs. (37):

$\chi_{\mathrm{s}} \simeq 4.0 \times L_{52}^{-1 / 12} r_{0,7}^{-1 / 12} \eta_{3}^{1 / 3}\left(\frac{1+\xi}{2}\right)^{1 / 3}$

For lower values of $\eta$, the saturation value $\chi_{\mathrm{s}}$ is smaller and reduces to the threshold values $\chi_{\mathrm{s}}=\chi_{\pi} \equiv 2.15$ when $\eta=\eta_{\pi}$. Adopting the value $\chi=\chi_{\mathrm{s}} \simeq 4$ we find from Eqs. (37), (52) and (55) that the neutrino energy in the observer frame can be expressed as

$\left\langle\epsilon_{v}\right\rangle=\frac{\alpha \Gamma_{\mathrm{np}} \epsilon_{v}^{0}}{1+z}$,

where $\alpha^{\mathrm{FB}} \simeq 4.5$ accounts for the non-zero kinetic energy of pions when they are created and for the fact that the particle distribution is not isotropic in the neutron rest frame. Using the same parameter range as in Sect. 3.2 we find from a numerical analysis that $\alpha^{\mathrm{FB}}$ should be slightly higher than this estimate and we will adopt $\alpha^{\mathrm{FB}} \simeq 6$ in the following.

In the AC model the situation is more complex because charged pions interact with the flow before decay and because pions are created at various radii in the flow. Since the pion gyration time is much shorter than both the synchrotron cooling time and their lifetime, pions will isotropize in the frame $K^{\prime}$ comoving with the proton fluid without significant energy loss. In this frame, the secondary pions are injected with energy $\left\langle\epsilon_{\pi}^{\prime}\right\rangle=\Gamma_{\mathrm{p}}^{\prime \prime}\left\langle\epsilon_{\pi}^{\prime \prime}\right\rangle$, where $\Gamma_{\mathrm{p}}^{\prime \prime}=\sqrt{s} /\left(2 m c^{2}\right)$ is the Lorentz factor of the incident proton as observed in the COM frame. The observed neutrino energy is then given by the following expression:

$\left\langle\epsilon_{v}^{\mathrm{AC}}\right\rangle=\frac{\Gamma_{\mathrm{p}} \Gamma_{\mathrm{p}}^{\prime \prime}}{1+z} \frac{\left\langle\epsilon_{\pi}^{\prime \prime}\right\rangle \epsilon_{v}^{0}}{m_{\pi} c^{2}} \simeq \frac{\Gamma_{\mathrm{np}}\left(\chi^{1 / 2}+\chi^{3 / 2}\right)}{2(1+z)} \frac{\left\langle\epsilon_{\pi}^{\prime \prime}\right\rangle \epsilon_{v}^{0}}{m_{\pi} c^{2}}$

where we approximate the proton and neutron dynamics by Eqs. (37) in the last equality. Note that the interaction with the flow results in a substantial increase in the observed energy of the secondary particles. 
For flows described by the AC model $n p$ collisions occur at various radii with different collision energies and different values for the Lorentz boost factor $\Gamma$. Therefore, we should average the observed energy given in Eq. (58) over the developing outflow. We express the probability for an interaction to occur while $\chi$ is in the range $\chi \ldots \chi+\mathrm{d} \chi$ as $\tau(\chi) \mathrm{d} \chi$. Since the scaling approximations (37) describe the flow around decoupling quite well in the AC model, we use Eq. (48) to estimate that

$\tau(\chi) \equiv \frac{\mathrm{d} \tau}{\mathrm{d} \chi}=\left(1-\frac{0.75}{\ln \chi}\right)\left(\chi^{-4}-\chi^{-6}\right)$.

Averaging Eq. (58) over this distribution we find that the observed neutrino energy can be expressed as in Eq. (57) with $\alpha^{\mathrm{AC}} \simeq 20$. This is in good agreement with numerical results in the same parameter range as in Sect. 3.2.

\subsection{Reprocessing of $\gamma$-rays: pair cascades versus synchrotron cooling}

While the flow is optically thin with respect to the emitted neutrinos resulting from charged pion decay, this is not the case for the $\gamma$-ray photons that are produced by neutral pion decay. In the proton rest frame, the $\gamma$-rays are injected with average energy (for a given value of $\chi$ )

$\left\langle\epsilon_{\gamma}^{\prime}\right\rangle=\frac{\Gamma_{\mathrm{p}}^{\prime \prime}\left\langle\epsilon_{\pi}^{\prime \prime}\right\rangle \epsilon_{\gamma}^{0}}{m_{\pi} c^{2}}$,

where $\epsilon_{\gamma}^{0}=70 \mathrm{MeV}$. Integrating over the developing flow as in the previous section, we express $\left\langle\epsilon_{\gamma}^{\prime}\right\rangle \simeq \beta \epsilon_{\gamma}^{0}$ and estimate analytically that $\beta \simeq 3$ for both the fireball and the AC model. This is consistent with numerical results. Hence $\gamma$-rays have a typical energy $\simeq 70 \beta \sim 200 \mathrm{MeV}$ in the proton rest frame and are ejected at radii $r \gtrsim r_{\pi}$; not far from the Thomson photosphere of the flow.

At these radii both fireballs and reconnection flows carry a soft photon field with characteristic comoving energy in the 1 keV range (see Derishev et al. 1999a; and Giannios 2006, for the fireball and reconnection model, respectively). Because of this intense soft photon field the flow is very optically thick with respect to these $\sim 200 \mathrm{MeV}$ photons, which are scattered and create pairs. In addition to the pairs that come from neutral pion decay, one energetic electron (or positron) is injected in the flow for every charged pion decay. We have included this contribution in the calculations that follow.

In the fireball model the dominant cooling mechanism of the electron-positron pair is inverse Compton scattering. The upscattered soft photons create more pairs resulting in pair cascades. In the reconnection model the flow is dominated by Poynting flux and the energy density of the magnetic field is much higher than the radiation energy density (see also Eq. (10) in Giannios 2006). As a result, the first generation of produced pairs cool down mainly through synchrotron emission. We discuss the outcome of the $\gamma$-ray injection separately for the two models.

\subsubsection{Pair cascades in fireball}

Photons in the fireball are upscattered by pairs and absorbed by soft photons during the pair cascade. In the case of a saturated cascade, where all upscattered photons are absorbed, about $\sim 10 \%$ of the energy of the $\gamma$-rays can be converted into rest mass of the pairs (Svensson 1987). More realistically the cascade is expected to be unsaturated, converting a few times less energy into rest mass of pairs (Derishev et al. 1999a; Belyanin et al. 2003).

The result of these pair cascades is twofold. Each injected $\gamma$-ray photon is reprocessed to multiple softer photons and the flow is loaded with pairs that contribute to its opacity. Although the saturation point of the cascade depends on the shape of the soft photon spectrum, we roughly estimate that photons with energies $\epsilon^{\prime} \sim 3 \mathrm{MeV}$ in the proton rest frame are able to escape (Belyanin et al. 2003). The overall emitted spectrum will be broad and most energy is emitted with observer energies in the range $\epsilon \sim \Gamma_{\mathrm{p}, \mathrm{s}}\left(\epsilon^{\prime} \ldots 10 \epsilon^{\prime}\right) /(1+z) \sim(2 \ldots 20) /(1+z) \mathrm{GeV}$. The strength of this component and its detection prospects are discussed in the next section.

To estimate the importance of pair loading in the flow, one should compare the number of produced pairs with the number of electrons (or, equivalently, protons) pre-existing in the flow. The flow has $\xi_{0}$ neutrons per proton out of which a fraction $\tau$ scatters inelastically. This results in $\xi_{0} \tau$ inelastic scatterings per proton. Every scattering results on average in $\sim 1 \gamma$-ray photon (see Eq. (51)) with a typical energy $70 \beta \mathrm{MeV}$ in the proton rest frame. A fraction $f \sim 3 \%$ of this energy is used in rest mass of pairs which results in $\sim 70 \beta f$ pairs per $\gamma$-ray.

By setting $\xi_{0}=1$ and using the values of $\beta$ and $\tau$ relevant for the reference values of the parameters for a fireball (see Sect. 3.2 and the beginning of this section), one finds that the pair cascades result in $\sim 0.15$ pairs per proton. For the neutrondominated case where $\xi_{0}=5$ we find significantly more pair loading, viz. $\sim 2$ pairs per proton. Note that we find significantly less pair loading of the flow because of pion decay compared to previous works. The main source for this discrepancy comes from the fact that, as we have shown in Sect. 3.2, the optical depth for inelastic $n p$ scattering is about one order of magnitude smaller than the value $\sim 1$ that is typically assumed in these studies.

Most of these pairs are produced at large radii - and low densities - so that they do not annihilate but stay in the flow. For $\xi_{0} \lesssim 1$, the contribution to the opacity from pair loading is at most moderate. On the other hand, for $\xi_{0} \gg 1$ the number of produced pairs exceeds that of the pre-existing electrons. A fraction of those are produced below the Thomson photosphere and its location is pushed to larger radii. This can have some backreaction on the dynamics of fireballs that can be accelerated to slightly higher bulk Lorentz factors than those calculated in Sect. 2.4.1, where this effect is neglected.

\subsubsection{Synchrotron cooling in the magnetized flow}

We now turn our attention to the reconnection model. The typical energy of the electron-positron pair produced by scattering of a $\gamma$-ray (resulting from neutral pion decay) with a soft photon is $\sim 120 \mathrm{MeV}$ which corresponds to a random electron Lorentz factor $\gamma_{\mathrm{e}} \simeq 200-300$. The produced pair finds itself in a strongly magnetized flow with comoving $B^{\prime} \simeq \sqrt{L / c r^{2} \Gamma_{\mathrm{p}}^{2}} \sim 10^{6} \mathrm{G}$ for typical values of the parameters and for the radii where most of the pion creation takes place.

Under these conditions, the synchrotron cooling timescale of the pair $t_{\mathrm{s}}^{\prime} \sim 10^{-6} \mathrm{~s}$ is much shorter than the Compton cooling timescale. The lack of pair cascades leads to negligible pair loading of the flow. The peak of the synchrotron emission is located at $\epsilon_{\mathrm{s}}^{\prime}=e \hbar B^{\prime} \gamma_{\mathrm{e}}^{2} / m_{\mathrm{e}} c \sim 0.2 \ldots 2 \mathrm{keV}$ in the proton rest frame. At the radii where most of the pion production takes place, the bulk Lorentz factor of the protons is $\Gamma_{\mathrm{p}} \sim 400-500$ which results in observer synchrotron peak in the sub-MeV energy range. 
Keeping the rest of the parameters fixed to their reference values, we find that the synchrotron emission peaks at observer energy $\epsilon_{\mathrm{s}} \simeq 120 \mathrm{keV}$ for $\xi_{0}=1$ and at $\epsilon_{\mathrm{s}} \simeq 600 \mathrm{keV}$ for $\xi_{0}=5$. The spectrum is characteristic of fast (synchrotron) cooling particles with an exponential cutoff above the peak and a low-energy spectral slope of $-1 / 2$. The strength of this component and its detection prospects are given in the next section.

\section{Detection prospects}

Using the results obtained in the previous section on the number and energy of secondary neutrinos and $\gamma$-rays created in inelastic $n p$ interactions, we discuss the detection prospects here.

\subsection{Neutrinos}

We express the neutrino fluence as observed on earth as

$\Phi_{v}=\frac{N_{\mathrm{n}} \mathcal{N}_{v} \mathcal{P}_{\mathrm{np}}}{4 \pi D_{\mathrm{p}}^{2}}$,

where $\mathcal{N}_{v}$ is the average number of neutrinos created per inelastic $n p$ interaction (we add the contribution of muon- and electron-(anti)neutrinos given in Eq. (51) here), $\mathcal{P}_{\mathrm{np}}$ is the inelastic $n p$ interaction probability, $D_{\mathrm{p}}$ is the proper distance, and

$N_{\mathrm{n}}=\frac{\xi_{0}}{1+\xi_{0}} \frac{E}{\eta m c^{2}}=3.3 \times 10^{52}\left(\frac{2 \xi_{0}}{1+\xi_{0}}\right) E_{53} \eta_{3}^{-1}$

denotes the number of neutrons contained in the outflow. In the last equation, $E$ denotes the total isotropic equivalent energy of the burst. Since $\mathcal{P}_{\mathrm{np}} \ll 1$ we express $\mathcal{P}_{\mathrm{np}} \simeq \tau$, where $\tau$ denotes the optical depth for inelastic $n p$ collisions.

We consider the optimistic case of a nearby energetic burst at redshift $z=0.1$. Assuming a universe that consists of matter and a cosmological constant, the proper distance $D_{\mathrm{p}}$ is given by the following expression:

$D_{\mathrm{p}}=\frac{c}{H_{0}} \int_{0}^{z} \frac{\mathrm{d} z^{\prime}}{\sqrt{\Omega_{\Lambda, 0}+\Omega_{m, 0}\left(1+z^{\prime}\right)^{3}}}$,

where $\Omega_{\Lambda, 0}$ and $\Omega_{\mathrm{m}, 0}$ denotes the current density parameters of the cosmological constant and matter, respectively, and $H_{0}$ is the Hubble parameter. Using the currently favored values $\Omega_{\Lambda, 0}=$ $0.76, \Omega_{\mathrm{m}, 0}=0.24$, and $H_{0}=73 \mathrm{~km} \mathrm{~s}^{-1} \mathrm{Mpc}^{-1}$ (Yao et al. 2006) we find a proper distance $D_{\mathrm{p}}=1.2 \times 10^{27} \mathrm{~cm}$. Inserting this in Eq. (68) we find the following neutrino particle fluences for the two models:

$\Phi_{v}^{\mathrm{FB}} \simeq 10^{-4}\left(\frac{\tau}{0.05}\right)\left(\frac{2 \xi_{0}}{1+\xi_{0}}\right) E_{53} \eta_{3}^{-1} \mathrm{~cm}^{-2}$

$\Phi_{v}^{\mathrm{AC}} \simeq 2 \times 10^{-5}\left(\frac{\tau}{0.01}\right)\left(\frac{2 \xi_{0}}{1+\xi_{0}}\right) E_{53} \sigma_{0,2}^{-3 / 2} \mathrm{~cm}^{-2}$.

As discussed in Sect. 3.2, a typical value for the inelastic $n p$ optical depth in the fireball model is $\tau^{\mathrm{FB}}=0.05$ and for the reconnection model $\tau^{\mathrm{AC}}=0.01$. The dependence on the model parameters, as obtained from a numerical analysis, is expressed in Eqs. (49) and (50).

From Eq. (57), the average neutrino energy as observed on earth is equal to

$\left\langle\epsilon_{v}\right\rangle=\frac{\alpha \Gamma_{\mathrm{np}} \epsilon_{v}^{0}}{1+z}$ where $\alpha$ is a numerical factor that accounts for the non-zero kinetic energy of pions when they are created and for the fact that the particle distribution is not isotropic in the neutron rest frame while we boost with $\Gamma_{\mathrm{np}}$ to the observer frame. Based on the results found in Sect. 3.4, we take $\alpha^{\mathrm{FB}}=6$ and $\alpha^{\mathrm{AC}}=20$ for the fireball model and the AC model, respectively. Using Eqs. (26) and (35) for the Lorentz factors at decoupling we find that $\left\langle\epsilon_{v}^{\mathrm{FB}}\right\rangle \simeq 50 \mathrm{GeV}$ and $\left\langle\epsilon_{v}^{\mathrm{AC}}\right\rangle \simeq 70 \mathrm{GeV}$ for reference values of the parameters and a burst at redshift $z=0.1$. These values depend only mildly on the parameters through the Lorentz factor at decoupling $\Gamma_{\mathrm{np}}$ but the value of $\alpha$ may change by a factor $\sim 2$ depending on the burst parameters.

Following Bahcall \& Mészáros (2000) we estimate the number of interactions $R_{v}$ in a large-volume neutrino detector due to the diffuse background as $R_{v}=\Phi_{v} \mathcal{R}_{\mathrm{b}} \sigma_{v} N_{\mathrm{t}}$, where $\mathcal{R}_{\mathrm{b}}=10^{3} \mathcal{R}_{\mathrm{b}, 3}$ denotes the burst rate per year, $\sigma_{v}=5 \times 10^{-39}\left(\epsilon_{v} / 1 \mathrm{GeV}\right) \mathrm{cm}^{2}$ is the neutrino interaction cross section and $N_{\mathrm{t}}=10^{39} N_{\mathrm{t}, 39}$ is the number of target protons in the detector. For reference values of the parameters and an average redshift $z=1$ we find that $R_{v}^{\mathrm{FB}} \simeq 0.3$ year $^{-1}$ and $R_{v}^{\mathrm{AC}} \simeq 0.07$ year $^{-1}$ for the fireball model and the AC model, respectively. Note that, for comparison with the literature, this estimate relies on the rather optimistic reference value of 1000 bursts per year leading to neutrinos through inelastic $n p$ collisions.

The predicted diffuse neutrino detection rate for the fireball model is a factor $\sim 5$ smaller than the results found by Bahcall \& Mészáros (2000). This is primarily due to the more accurate cross sections used in this work and the distinction between $n p$ decoupling radius and pion creation radius. This distinction also implies that the condition for inelastic $n p$ collisions to occur (as expressed in Eq. (43)) is more stringent than the condition presented by Bahcall \& Mészáros (2000). Therefore, the fraction of GRBs for which $n p$ decoupling occurs is expected to be much smaller and the reference value $\mathcal{R}_{\mathrm{b}}=10^{3}$ is not very realistic. For the reconnection model, we find that the expected neutrino fluence is typically lower than those for the fireball model by a factor $\sim 5$. This results from the fact that the pion production radius is much larger than the $n p$ decoupling radius, which is a very robust feature of this model. The condition for inelastic $n p$ collisions as expressed in Eq. (45), on the other hand, is fulfilled in a large range of the parameters of the model. It is therefore expected that $n p$ decoupling occurs in a large fraction of GRBs for the reconnection model.

\subsection{Gamma rays}

Secondary $\gamma$-rays resulting from $n p$ collisions are reprocessed by the flow due to interactions with the soft photon field (see Sect. 3.5). This results in pair cascades for fireballs and in electron synchrotron emission for AC outflows. The total energy (in the frame of the progenitor) that is injected in the flow in the form of $\gamma$-rays is equal to

$E_{\gamma}=\frac{\Gamma_{\mathrm{p}} \Gamma_{\mathrm{p}}^{\prime \prime} N_{\mathrm{n}} \tau\left\langle\epsilon_{\pi}^{\prime \prime}\right\rangle \epsilon_{\gamma}^{0}}{m_{\pi} c^{2}}=\gamma \Gamma_{\mathrm{np}} N_{\mathrm{n}} \epsilon_{\gamma}^{0}$,

which defines the factor $\gamma$. We find that $\gamma \simeq 0.5$ for both the fireball model (for $\eta \sim$ few $\eta_{\pi}$ ) and the AC model (for $\sigma_{0} \sim$ few $\left.\sigma_{0, \pi}\right)$. For reference values of the parameters this implies that the fraction of the burst energy that is converted to $\gamma$-rays is roughly $5 \times 10^{-3}$ for fireballs and roughly $2 \times 10^{-3}$ for the AC model. We assume that the bulk of the energy given in Eq. (67) leaves the source after reprocessing, albeit in photons of lower energies. 


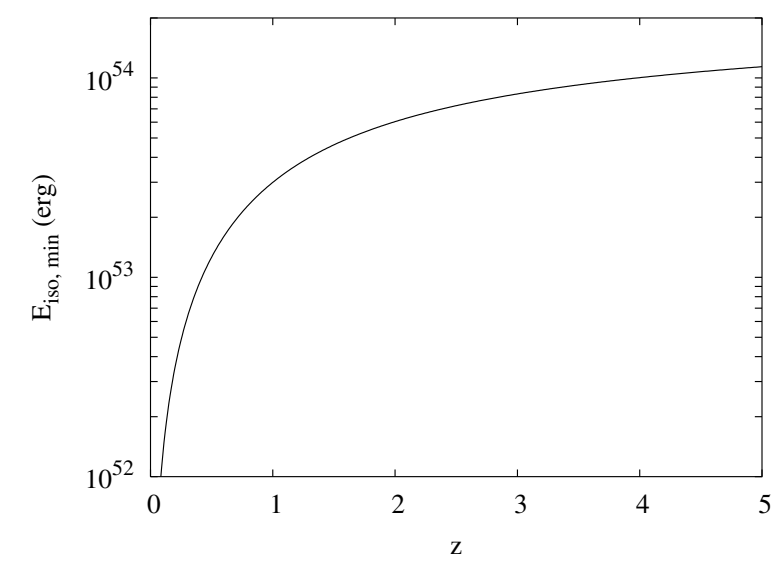

Fig. 5. Minimum value for the total isotropic burst energy $E_{\text {iso }}$ for which the reprocessed $\gamma$-ray emission (in the fireball model) is above the GLAST threshold, as a function of redshift. In this figure we have taken $\xi_{0}=r_{0,7}=\eta_{3}=1$, and we have taken the burst duration equal to $10 \mathrm{~s}$.

In Sect. 3.5.1 we estimated that the $\gamma$-ray emission from pair cascades in the fireball model is in the range $2-20 \mathrm{GeV}$ in the frame of the progenitor. From this we estimate the $\gamma$-ray number fluence $\Phi_{\gamma}$ from a source at proper distance $D_{\mathrm{p}}$ as

$\Phi_{\gamma}=\frac{E_{\gamma}}{4 \pi D_{\mathrm{p}}^{2} \epsilon_{\gamma}^{\text {casc }}}$,

where $\epsilon_{\gamma}^{\text {casc }} \simeq 10 \mathrm{GeV}$ is the average $\gamma$-ray energy emitted by the pair cascades. For an energetic burst at $z=0.1$ the number fluence is $\Phi_{\gamma} \simeq 10^{-3} \mathrm{~cm}^{-2}$ which can be detected with the upcoming GLAST satellite that has an effective area $\sim 10^{4} \mathrm{~cm}^{2}$ at these energies (Gehrels \& Michelson 1999). In fact, we find that this emission is detectable for a fairly large range of parameters. In Fig. 5 we indicate, as a function of redshift, the minimum total isotropic burst energy for which the gamma-ray emission by this mechanism is detectable with GLAST. In producing this figure we have chosen reference values for the relevant model parameters and assumed a burst duration of $10 \mathrm{~s}$.

The isotropic equivalent energy carried by the prompt emission at $\sim \mathrm{MeV}$ energies of a typical GRB is in the range $10^{52}-10^{54} \mathrm{erg}$. This is only a lower limit for the isotropic equivalent energy of the ultrarelativistic flow which may well be a factor $\sim 10$ larger than the energy carried by the prompt emission, depending on the unknown efficiency of the mechanism that generates the prompt emission. Therefore the minimum energy shown in Fig. 5 is not very restrictive and we expect that this emission is detectable for a fairly large fraction of GRBs in which protons and neutrons decouple. This conclusion also holds for high redshifts where the volume for GRBs to occur is largest.

Apart from the collisions between bulk protons and neutrons considered in this work, pions can also be created by nuclear collisions as a result of internal shocks in the sub-photospheric region of the flow (Mészáros \& Rees 2000). This mechanism can inject $\gamma$-rays in the flow in a different region of the GRB parameter space.

In the AC model the energy is radiated as synchrotron emission with energy of a few hundred $\mathrm{keV}$ in the observer frame (see Sect. 3.5.2). The corresponding energy fluence $\sim 10^{-5} \mathrm{erg} \mathrm{cm}^{-2}$ is lower than the expected prompt emission for a burst at $z=$ 0.1 with the reference values adopted here and for a typical prompt emission radiative efficiency $\gtrsim 0.1$. This makes it very hard to disentangle this $\gamma$-ray signal from the prompt emission.
Of course this conclusion holds as long as the energy of the reprocessed $\gamma$-rays is much less than the energy of the prompt emission and the radiative efficiency for the prompt emission is larger than the energy fraction $\sim 2 \times 10^{-3}$ transferred to $\gamma$-rays by $n p$ collisions in the AC model. On the other hand, even though this synchrotron component is in general weak, it may have a substantial contribution to the prompt X-ray emission since its flux increases with decreasing energy as $f_{v} \sim v^{-1 / 2}$ (i.e., following the characteristic slope of fast-cooling synchrotron emission).

\section{Conclusion}

In this work we have found that $\gamma$-ray emission resulting from inelastic collisions between differentially streaming neutrons and protons and reprocessed by the flow may be a useful diagnostic of the nature of GRB outflows. Provided that the baryon loading of the flow is sufficiently small, a few per mille of the burst energy is reinjected in the flow through $n p$ collisions in both the fireball model and in the AC model, which was used in this work as a specific model for GRB flows that are powered by magnetic reconnection. In the fireball model, the injection of these $\gamma$-rays in the outflow leads to pair cascades and subsequently to the emission of $\gamma$-rays with observer energy in the range of $2-20 \mathrm{GeV} /(1+z)$. In Fig. 5, we show the minimum total isotropic burst energy, as a function of redshift, for which this emission can be detected by GLAST. The constraint on the energy is not very restrictive and hence this $\gamma$-ray emission should be detectable for a fairly large fraction of the GRBs in which $n p$ decoupling occurs. In the AC model, synchrotron energy loss prevents pair cascading and the energy is radiated away at much lower observer energies of a few hundred $\mathrm{keV}$. This component is expected to be dominated by the prompt $\gamma$-ray emission.

The neutrino particle fluence from $\pi^{ \pm}$decay created in inelastic $n p$ collisions in the fireball model is found to be an order of magnitude smaller than previous estimates. This is due to the more accurate cross sections for elastic and inelastic $n p$ scattering used in this work and the distinction between $n p$ decoupling radius and the pion production radius. The neutrino fluence in the $\mathrm{AC}$ model is smaller by another factor $\sim 5$ due to the very gradual acceleration of the flow, which is a very robust feature of the model. The energy of neutrinos from $n p$ interactions in GRB outflows as observed on earth is in the range 50-70 GeV for reference values of the parameters, which is somewhat higher than previous estimates. We find that the observed neutrino energy in the AC model is higher than in the fireball model because the strong magnetic field causes the charged pions to isotropize in the proton rest frame rather than in the collision COM frame. Unfortunately the neutrino emission in both models is so low that it is very difficult to use its properties to constrain the physics of GRB outflows.

In both the fireball model and the $\mathrm{AC}$ model we find that inelastic $n p$ collisions occur only if the baryon loading is sufficiently low (see Sect. 3.1). For the fireball model, this condition is quite restrictive and we expect that inelastic $n p$ collisions are only possible for exceptional bursts. On the other hand, inelastic collisions occur for a large range of the parameters in the reconnection model.

The above results rely on a proper understanding of the dynamics of the flow. We have discussed the effect of neutrons on the dynamics of the flow in Sect. 2 (some numerical results are presented in Figs. 1-4). We present a numerical model which includes the acceleration of the protons due to energy conversion in the flow, coupling of neutrons to protons by nuclear scattering 
(and the dynamical decoupling of neutrons and protons) and neutron decay. To a first approximation the dynamics of protons and neutrons can be described by the analytical model given in Eqs. (37). This model provides a useful estimate for the $n p$ inelastic optical depth (Sect. 3.2) and the energies of neutrinos (Sect. 3.4) and $\gamma$-rays (Sect. 3.5). These estimates are generally in good agreement with results obtained from the numerical model described in Sect. 2 (some differences are discussed in the main text). The analytical estimates can be extended in a straightforward manner to any flow with $\Gamma_{\mathrm{p}} \propto r^{p}$ and $\Gamma_{\mathrm{n}}=$ const.

From an observational point of view, the most promising conclusion of this work is that $\gamma$-ray emission resulting from $n p$ interactions may provide a signature of the nature of the flow (Sect. 3.5). The difference in energy of the reprocessed $\gamma$-ray emission between the fireball model and the AC model results essentially from the difference in the ratio of magnetic energy density to radiation energy density. Therefore the energy of this emission appears to be a robust probe for the physics of GRB outflows. In this work we have estimated the $\gamma$-ray energy and fluence for reference values of the burst parameters. A more detailed analysis is necessary to study the spectral properties of the emission and compare it with other emission mechanisms over a broad range of parameters.

It was pointed out recently that a substantial neutron component in GRB flows may affect the properties of GRB afterglows (Belobororov 2003a). This provides a way of constraining the physics of GRB outflows from afterglow observations. The numerical model discussed in this work can be used to study this possibility in more detail. Another interesting question is whether inhomogeneities in the flow can cause significant particle production through $n p$ collision in the $\mathrm{AC}$ model (for fireballs, this was discussed by Mészáros \& Rees 2000). These issues are left for future work.

Acknowledgements. H.K. wishes to thank the Max Planck Institute for Astrophysics, where part of this work was completed, for its hospitality. H.K. acknowledges many valuable discussions with Ralph Wijers and Asaf Pe'er. D.G. thanks the Astronomical Institute "Anton Pannekoek" for its hospitality during the initiation of this work. We thank the referee for useful comments that helped to improve the quality of this paper.

\section{Appendix A: Cross section approximations}

In this work we use the following approximations for the total and elastic $n p$ cross sections:

$\sigma_{\text {tot }}=\max \left[\frac{\bar{\sigma}}{0.19 \beta_{\text {rel }}+5.2 \beta_{\text {rel }}^{3}}, \bar{\sigma}\right] ;$

$\sigma_{\mathrm{el}}\left(\chi \geq \chi_{\pi}\right)=\frac{0.75 \bar{\sigma}}{\ln \chi}$

where $\bar{\sigma} \equiv 4 \times 10^{-26} \mathrm{~cm}^{2}$ and $\chi \equiv \Gamma_{\mathrm{p}} / \Gamma_{\mathrm{n}}$. At energies below the pion production threshold $\left(\chi<\chi_{\pi}\right)$ the elastic cross section $\sigma_{\mathrm{el}}=$ $\sigma_{\text {tot }}$. Hence the inelastic cross section above the pion production threshold can be approximated with:

$\sigma_{\text {inel }}\left(\chi \geq \chi_{\pi}\right)=\sigma_{\text {tot }}-\sigma_{\text {el }}=\bar{\sigma}\left(1-\frac{0.75}{\ln \chi}\right)$.

In these equations, $\beta_{\text {rel }}$ and $\chi$ are related to the incident proton momentum in the neutron rest frame $p_{\mathrm{p}}^{\prime}$ as follows:

$\beta_{\text {rel }} \equiv \frac{p_{\mathrm{p}}^{\prime}}{\sqrt{{p_{\mathrm{p}}^{\prime 2}+m^{2} c^{2}}^{2}}}$

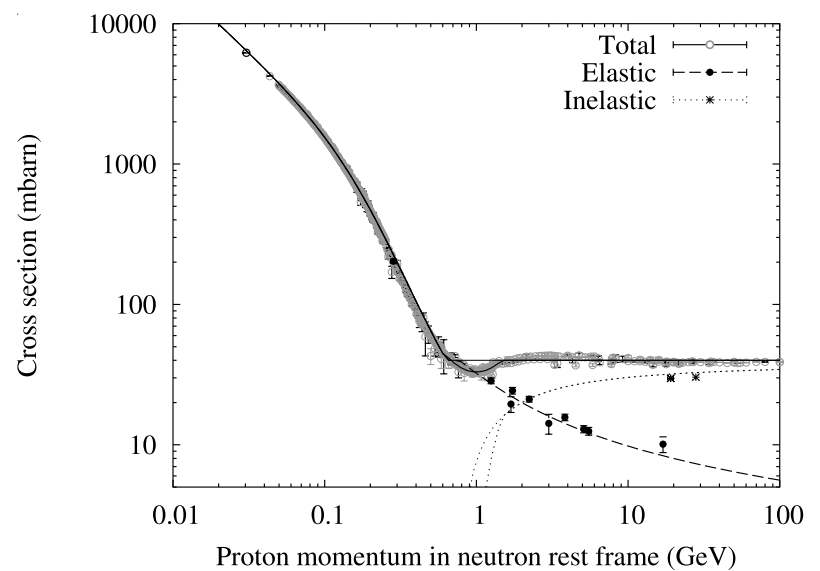

Fig. A.1. Experimental data and approximations of the total, elastic and inelastic $n p$ cross sections. The thin lines show the approximations used for the analytical model; the thick lines show those used in the numerical computation.

$\chi \equiv \frac{\Gamma_{\mathrm{p}}}{\Gamma_{\mathrm{n}}}=\frac{p_{\mathrm{p}}^{\prime}}{m c}+\sqrt{\frac{p_{\mathrm{p}}^{\prime 2}}{m^{2} c^{2}}+1 .}$

The approximations given in Eqs. (A.1)-(A.3) are shown in Fig. A.1, together with experimental data (Yao et al. 2006) and the approximation that was used to describe the inelastic cross section in the numerical analysis.

\section{Appendix B: Frames and Lorentz factors}

The Lorentz factor of protons and neutrons in the observer frame $K$ are denoted with $\Gamma_{\mathrm{p}}$ and $\Gamma_{\mathrm{n}}$, respectively, and we assume that both $\Gamma_{\mathrm{p}} \gg 1$ and $\Gamma_{\mathrm{n}} \gg 1$. In the observer frame, the COM frame $K^{\prime \prime}$ of the $n p$ collision is moving with Lorentz factor

$\Gamma_{\mathrm{COM}}=\sqrt{\Gamma_{\mathrm{p}} \Gamma_{\mathrm{n}}}$

In the COM frame, protons and neutrons are moving in opposite directions with Lorentz factors

$\Gamma_{\mathrm{p}}^{\prime \prime}=\Gamma_{\mathrm{n}}^{\prime \prime}=\frac{\sqrt{s}}{2 m c^{2}}=\frac{1}{2}\left(\frac{\Gamma_{\mathrm{p}}}{\Gamma_{\mathrm{n}}}\right)^{1 / 2}+\frac{1}{2}\left(\frac{\Gamma_{\mathrm{n}}}{\Gamma_{\mathrm{p}}}\right)^{1 / 2}$,

where we take the proton and neutron masses equal to $m$. In the main text we use $K^{\prime}$ to denote the rest frame of either the proton or the neutron. If $K^{\prime}$ denotes the proton rest frame, $\Gamma_{\mathrm{p}}^{\prime}=1$ by definition and

$\Gamma_{\mathrm{n}}^{\prime}=\frac{1}{2}\left(\frac{\Gamma_{\mathrm{p}}}{\Gamma_{\mathrm{n}}}+\frac{\Gamma_{\mathrm{n}}}{\Gamma_{\mathrm{p}}}\right)=2\left(\Gamma_{\mathrm{n}}^{\prime \prime}\right)^{2}-1$

\section{References}

Bahcall, J. N., \& Mészáros, P. 2000, PRL, 85, 1362

Beloborodov, A. M. 2002, ApJ, 565, 808

Beloborodov, A. M. 2003a, ApJ, 585, L19

Beloborodov, A. M. 2003b, ApJ, 588, 931

Belyanin, A. A., et al. 2003, in Gamma-Ray Burst and Afterglow Astronomy 2001: A Workshop Celebrating the First Year of the HETE Mission, ed. G. R. Ricker, \& R. K. Vanderspek, AIP Conf. Proc., 662

Cavallo, G., \& Rees, M. J. 1978, MNRAS, 183, 359

Chen, W. X., \& Beloborodov, A. M. 2007, ApJ, 657, 383

Coroniti, F. V. 1990, ApJ, 349, 538

Daum, M., Finger, M., Finger, M. Jr., et al. 2002, Eur. Phys. J., C23, 43

Derishev, E. V., Kocharovksy, V. V., \& Kocharovksy, V1. V. 1999a, ApJ, 521, 640 
Derishev, E. V., Kocharovksy, V. V., \& Kocharovksy, Vl. V. 1999b, A\&A, 345, L51

Drenkhahn, G. 2002, A\&A, 387, 714

Drenkhahn, G., \& Spruit, H. C. 2002, A\&A, 391, 1141

Fan, Y. Z., \& Wei, D. M. 2004, ApJ, 615, L69

Fan, Y. Z., Zhang, B., \& Wei, D. M. 2005, ApJ, 628, 298

Gehrels, N., \& Michelson, P. 1999, Astropart. Phys., 11, 277

Giannios, D. 2006, A\&A, 457, 763

Giannios, D., \& Spruit, H. C. 2005, A\&A, 430, 1

Giannios, D., \& Spruit, H. C. 2007, A\&A, 469, 1

Goodman, J. 1986, ApJ, 308, L47

Inoue, S., Iwamoto, N., Orito, M., Terasawa, M., et al. 2003, ApJ, 595, 294

Kleinschmidt, M., et al. 1980, Z. Phys. A, 298, 253

Landau, L. D., \& Lifshitz, E. M. 1971, The Classical Theory of Fields, Pergamon Press

Lyubarsky, Y. E. 2005, MNRAS, 358, 113

Lyubarsky, Y., \& Kirk, J. G. 2001, ApJ, 547, 437

Lyutikov, M. 2006, New J. Phys., 8, 119

Lyutikov, M., \& Blandford, R. D. 2003 [arXiv: astro-ph/0312347]

Mészáros, P. 2006, Rep. Prog. Phys., 69, 2259

Mészáros, P., \& Rees, M. J. 1997, ApJ, 482, L29

Mészáros, P., \& Rees, M. J. 2000, ApJ, 541, L5
Paczyński, B. 1986, ApJ, 308, L43

Peng, F., Königl, A., \& Granot, J. 2005, ApJ, 626, 966

Piran, T. 2004, Rev. Mod. Phys., 76, 1143

Piran, T., Shemi, A., \& Narayan, R. 1993, MNRAS, 263, 861

Prokoshin, Iu. D., \& Tiapkin, A. A. 1957, Sov. Phys. JETP, 5, 618

Pruet, J., Woosley, S. E., \& Hoffman, R. D. 2003, ApJ, 586, 1254

Razzaque, S., \& Mészáros, P. 2006, ApJ, 650, 998

Rossi, E. M., Beloborodov, A. M., \& Rees, M. J. 2006, MNRAS, 369, 1797

Spruit, H. C., Daigne, F., \& Drenkhahn, G. 2001, A\&A, 369, 694

Svensson, R. 1987, MNRAS, 227, 403

Thompson, C. 1994, MNRAS, 270, 480

Usov, V. V. 1992, Nature, 357, 472

Uzdensky, D. A., \& MacFadyen, A. I. 2006, ApJ, 647, 1192

Van Paradijs, J., Kouveliotou, C., \& Wijers, R. A. M. J. 2000, ARA\&A, 38, 379

Van Putten, M. H. P. M., \& Ostriker, E. C. 2001, ApJ, 552, L31

Vlahakis, N., \& Königl, A. 2001, ApJ, 563, L129

Vlahakis, N., \& Königl, A. 2003, ApJ, 596, 1080

Vlahakis, N., Peng, F., \& Königl, A. 2003, ApJ, 594, L23

Waxman, E., \& Bahcall, J. 1997, PRL, 78, 2292

Woosley, S. E., \& Bloom, J. S. 2006, ARA\&A, 44, 507

Yao, W.-M., et al. 2006, J. Phys. G, 33, 1 